\title{
Far-infrared and molecular line observations of Lynds 183 - Studies of cold gas and dust ${ }^{\star}$
}

\author{
M. Juvela ${ }^{1}$, K. Mattila ${ }^{2}$, K. Lehtinen ${ }^{2}$, D. Lemke ${ }^{3}$, R. Laureijs ${ }^{4}$, and T. Prusti ${ }^{5}$ \\ 1 Helsinki University Observatory, Tähtitorninmäki, PO Box 14, SF-00014 University of Helsinki, Finland \\ 2 Helsinki University Observatory, Tähtitorninmäki, PO Box 14, SF-00014 University of Helsinki, Finland \\ 3 Max-Planck-Institut für Astronomie, Königstuhl 17, 69117 Heidelberg, Germany \\ 4 ISO Data Centre, Astrophysics Division, Space Science Department of ESA, Villafranca del Castillo, \\ PO Box 50727, 28080 Madrid, Spain \\ 5 Astrophysics Division, Space Science Department of ESA, ESTEC, PO Box 299, 2200 AG Noordwijk, \\ The Netherlands
}

Received 25 september 2001 / Accepted 29 October 2001

\begin{abstract}
We have mapped the dark cloud L183 in the far-infrared at $100 \mu \mathrm{m}$ and $200 \mu \mathrm{m}$ with the ISOPHOT photometer aboard the ISO satellite. The observations make it possible for the first time to study the distribution and properties of the large dust grains in L183 without confusion from smaller grains. The observations show clear colour temperature variations which are likely to be caused by changes in the emission properties of the dust particles. In the cloud core the far-infrared colour temperature drops below $12 \mathrm{~K}$. The data allow a new determination of the cloud mass and the mass distribution based on dust emission. The estimated mass within a radius of $10^{\prime}$ from the cloud centre is $25 M_{\odot}$. We have mapped the cloud in several molecular lines including $\mathrm{DCO}+(2-1)$ and $\mathrm{H}^{13} \mathrm{CO}+(1-0)$. These species are believed to be tracers of cold and dense molecular material and we detect a strong anticorrelation between the $\mathrm{DCO}+$ emission and the dust colour temperatures. In particular, the $\mathrm{DCO}+(2-1)$ emission is not detected towards the maximum of the $100 \mu \mathrm{m}$ emission where the colour temperature rises above $15 \mathrm{~K}$. The $\mathrm{H}^{13} \mathrm{CO}+$ emission follows closely the $\mathrm{DCO}+$ distribution but $\mathrm{CO}$ isotopes show strong emission even towards the $100 \mu \mathrm{m}$ peak. Detailed comparison of the $\mathrm{DCO}+$ and $\mathrm{C}^{18} \mathrm{O}$ maps shows sharp variations in the relative intensities of the species. Morphologically the $200 \mu \mathrm{m}$ dust emission traces the distribution of dense molecular material as seen e.g. in $\mathrm{C}^{18} \mathrm{O}$ lines. A comparison with dust column density shows, however, that $\mathrm{C}^{18} \mathrm{O}$ is depleted by a factor of $\sim 1.5$ in the cloud core. We present results of $R$ - and $B$-band starcounts. The extinction is much better correlated with the $200 \mu \mathrm{m}$ than with the $100 \mu \mathrm{m}$ emission. Based on the $200 \mu \mathrm{m}$ correlation at low extinction values we deduce a value of $\sim 17^{\mathrm{m}}$ for the visual extinction towards the cloud centre where no background stars are observed anymore.
\end{abstract}

Key words. ISM: clouds - ISM: molecules - infrared: ISM: continuum - radio lines: ISM - radiative transfer ISM: individual objects: L183, L134N

\section{Introduction}

The distribution of material in dense interstellar clouds can be traced by either molecular line emission or the continuum emission from dust particles. The analysis of line spectra is complicated by radiative transfer effects. The emission of radiation depends on local properties like density and kinetic temperature. Based on these parameters, the observed line intensities can be predicted with radiative transfer modelling. In practice, the density structure

Send offprint requests to: M. Juvela, e-mail: mjuvela@astro.helsinki.fi

* Based on observations with ISO, an ESA project with instruments funded by ESA Member States (especially the PI countries: France, Germany, The Netherlands and the UK) and with the participation of ISAS and NASA. of the sources is, however, unknown and e.g. small scale density and velocity variations can seriously affect the observed intensities. Secondly, the ratio between the abundance of the tracer and the total gas mass is only known approximately and it can change even within one source. The chemical reactions depend on density and temperature (Millar et al. 1997; Lee et al. 1996) and therefore the chemistry of a dark cloud cores will differ from the chemistry in the surrounding warmer material.

Molecular abundances depend on the strength of the ultraviolet radiation field via processes such as photodissociation. The radiation is weakened by dust extinction and by the self-shielding of the molecules and this introduces radial abundance gradients. Selective photo-dissociation can lead to strong abundance variations between different $\mathrm{CO}$ isotopes and can even affect the excitation of the 
molecules (Warin et al. 1996) The abundances change even as a function of time (Leung et al. 1984; Herbst \& Leung 1989, 1990; Bettens et al. 1995) and the observed abundances have been used as a "chemical clock" to determine the evolutionary stage of sources (e.g. Stahler 1984; Lee et al. 1996). Observations of e.g. TMC-1 show clear anticorrelation between carbon bearing and oxygen bearing molecules (Pratap et al. 1997). This can be compared with models (e.g. Bergin et al. 1995, 1996) which show that CS and long carbon chain molecules are produced preferentially at early times, while SO abundance rises only much later.

The role of dust grains in chemical evolution is important but not yet well understood. In dark cores some molecules can freeze onto the dust grains and this causes the under-abundance of the molecules in the gas phase. CO abundances can decrease by more than a factor of ten (Gibb \& Little 1998). In the cold cores, the abundance of deuterated species, e.g. DCO+ are enhanced. This fact has been used to identify condensations in the earliest stages of star formation, before the birth of protostars again increases the temperatures (e.g. Loren et al. 1990). The abundance ratio $[\mathrm{DCO}+] /[\mathrm{HCO}+]$ depends also on the abundance of free electrons and can therefore also be used to determine this quantity (e.g. Guélin et al. 1977, 1982; Watson 1978; Wootten 1982; Anderson et al. 1999). The electron abundance is an important parameter since, through ambipolar diffusion, it affects the timescales of physical cloud evolution and eventually the rate of the star formation (Caselli et al. 1998).

The analysis of far-infrared dust continuum observations is in principle more straightforward. The emission is optically thin and the intensity directly related to the dust column density. At wavelengths $100-200 \mu \mathrm{m}$ the radiation is emitted mainly by the classical large grains, with a possible small contribution at $100 \mu \mathrm{m}$ by the "very small grains". The large grains heated by the normal interstellar radiation field settle to a temperature of $\sim 17 \mathrm{~K}$ and the maximum of the spectral energy distribution is close to $200 \mu \mathrm{m}$. The temperature of the small grains fluctuates and most of the radiation is at shorter wavelengths $(\lambda<100 \mu \mathrm{m})$. In the model of Désert et al. (1990) some $10 \%$ of the infrared emission at $100 \mu \mathrm{m}$ comes from very small dust grains.

The large grains are well mixed with the gas phase (Bohlin et al. 1978) and the FIR emission can be used to trace the gas distribution. The dust emission corresponding to a given column density varies depending on the composition and temperature of the dust. The farinfrared colour temperature has been observed to drop towards the centre of many dark cores (e.g. Clark et al. 1991; Laureijs et al. 1991) and even cirrus type clouds (Bernard et al. 1999). It is clear that the variations cannot be entirely due to a reduced radiation field, i.e. the observed colour temperature variations indicate a change in the grain properties. In the cloud cores, the dust particles may grow through coagulation, and in this case the associated change in the grain emissivity could explain part of the colour temperature variations (e.g. Meny et al. 2000; Cambrésy et al. 2001). Changes in the dust properties cause uncertainties to the column density estimates. These are, however, smaller than those associated with the analysis of optically thick molecular lines.

Most of the listed problems can be addressed by comparing observations of molecular line emission with farinfrared dust emission. In this paper we present such a study of the dark cloud L183.

\section{1. $L 183$}

L183 (L134N) is a well known dark cloud belonging to a high latitude cloud complex (Galactic coordinates $(l, b)=(6.0,36.7))$. The distance of the cloud has been estimated to be 100 pc (Mattila 1979; Franco 1989) although a distance of $160 \mathrm{pc}$ has also been used (Snell 1981). We adopt $d=100$ pc. Extinction towards the centre of L183 is at least $A_{V} \sim 6$ (Laureijs et al. 1991) and may exceed $A_{V}=10$. The cloud core is surrounded by an extended low extinction $\left(A_{V}<1\right)$ envelope.

L183 was observed in $\mathrm{CO}(1-0)$ and ${ }^{13} \mathrm{CO}(1-0)$ by Snell (1981), in $\mathrm{NH}_{3}$ by Ungerechts et al. (1980) and in several lines including $\mathrm{C}^{18} \mathrm{O}(1-0)$ and $\mathrm{H}^{13} \mathrm{CO}+(1-0)$ by Swade (1989a, 1989b). The ${ }^{13} \mathrm{CO}$ line map shows a cometary shape with the head of the globule pointing north in equatorial coordinates ( galactic east). This is also the densest part of the cloud. The cloud has a sharp edge towards the north, but in the southern part the strong ${ }^{13} \mathrm{CO}$ and especially the ${ }^{12} \mathrm{CO}$ emission continues in the tail that points towards L1780. Together, the region containing clouds L134, L169, L183, L1780 is sometimes called the $\mathrm{L} 134$ complex. $\mathrm{NH}_{3}$ and $\mathrm{C}_{3} \mathrm{H}_{2}$ emission is concentrated into the head region and ammonia emission is restricted to a very narrow ridge running from south to north. The usual centre position given for the cloud, $\left(\mathrm{RA}=15^{\mathrm{h}} 51^{\mathrm{m}} 30^{\mathrm{s}}\right.$ Dec $\left.=-2^{\circ} 43^{\prime} 31^{\prime \prime}, 1950.0\right)$ is close to the centre of the ammonia emission where density reaches a few times $10^{4} \mathrm{~cm}^{-3}$ (Swade 1989b). In recent years the cloud has been observed in a number of molecular lines (see e.g. Swade \& Schloerb 1992; Turner et al. 1999, 2000; Dickens et al. 2000; references therein).

The infrared emission from L183 was studied by Laureijs et al. $(1991,1995)$ based on the IRAS data. The $60 \mu \mathrm{m}$ emission is detected only in a narrow layer surrounding the cloud core. Based on the upper limit of the $I(60 \mu \mathrm{m}) / I(100 \mu \mathrm{m})$ ratio, an upper limit of $15 \mathrm{~K}$ was derived for the colour temperature assuming a $\nu^{2}$ emissivity law. However, at $60 \mu \mathrm{m}$, most of the emission comes from small dust particles, and the colour temperature is not directly related to the physical grain temperature. In the cloud core the lack of $60 \mu \mathrm{m}$ emission was interpreted as evidence for mantle growth. Ward-Thompson et al. (1994, 2000) have made sub-millimetre continuum observations of the cloud and have detected two point sources close to the cloud centre. Lehtinen et al. (2000) have studied the 
properties of the sources using far-infrared observations made with ISO.

In this paper we study the dark cloud L183 using $100 \mu \mathrm{m}$ and $200 \mu \mathrm{m}$ maps and new molecular line observations. The far-infrared observations were carried out with the ISOPHOT instrument aboard the ISO satellite. Both $100 \mu \mathrm{m}$ and $200 \mu \mathrm{m}$ are dominated by emission from classical large grains and this makes it possible, for the first time, to study the variations in the properties of the large grains without confusion from smaller size dust populations. The far-infrared observations enable us to determine the mass distribution of L183 independently from the molecular line data which may be affected by chemical fractionation and other systematic effects. The dust distribution will be compared with molecular line observations which include new ${ }^{12} \mathrm{CO},{ }^{13} \mathrm{CO}$ and $\mathrm{C}^{18} \mathrm{O}$ observations that cover the entire cloud L183. The correlations with dust column densities will be used to determine the degree of $\mathrm{C}^{18} \mathrm{O}$ depletion in the core of L183. New DCO+(2-1) and $\mathrm{H}^{13} \mathrm{CO}+(1-0)$ mappings have been made with higher spatial resolution than what were previously available. $\mathrm{DCO}+$ is known to be a tracer of cold gas and we will determine the correlations between $\mathrm{DCO}+$ and dust emission, especially the FIR colour temperatures. We will also study the variations in the intensity ratio of $\mathrm{DCO}+$ and $\mathrm{H}^{13} \mathrm{CO}+$. Finally, we present results of $R$ - and $B$-band star counts and a comparison between the extinction and the far-infrared emission.

\section{Observations and data processing}

\subsection{FIR observations}

Far-infrared observation of L183 were done with the ISOPHOT photometer (Lemke et al. 1996) aboard the ISO satellite (Kessler et al. 1996). Two $30^{\prime} \times 30^{\prime}$ maps were made in the PHT22 staring raster map mode with filters C_100 and C_200. The reference wavelengths of the filters are $100 \mu \mathrm{m}$ and $200 \mu \mathrm{m}$ and the respective pixel sizes $\sim 44^{\prime \prime}$ and $89^{\prime \prime}$.

Additional narrow strips running from NW towards the cloud centre were observed at $80 \mu \mathrm{m}, 100 \mu \mathrm{m}, 120 \mu \mathrm{m}$, $150 \mu \mathrm{m}$ and $200 \mu \mathrm{m}$. The analysis of these observations will be presented by Lehtinen et al. (in preparation).

Comparison of the ISOPHOT $100 \mu \mathrm{m}$ observations with the $100 \mu \mathrm{m}$ IRAS ISSA maps showed peculiar scatter with two separate branches in the ISOPHOT vs. IRAS relation. The calibration of the ISOPHOT observations was done with Fine Calibration Source (FCS) measurements that were done before and after the actual mapping and a linear interpolation was performed to determine the detector responsivity at each map position. The detector signals did not, however, stabilize during FCS measurements and this leads to some uncertainty. A $5 \%$ change in the relative responsivities of the two FCS measurements was found to be enough to minimize the scatter relative to the IRAS map. The average responsivity over the map was not changed. The resulting relation between ISOPHOT and

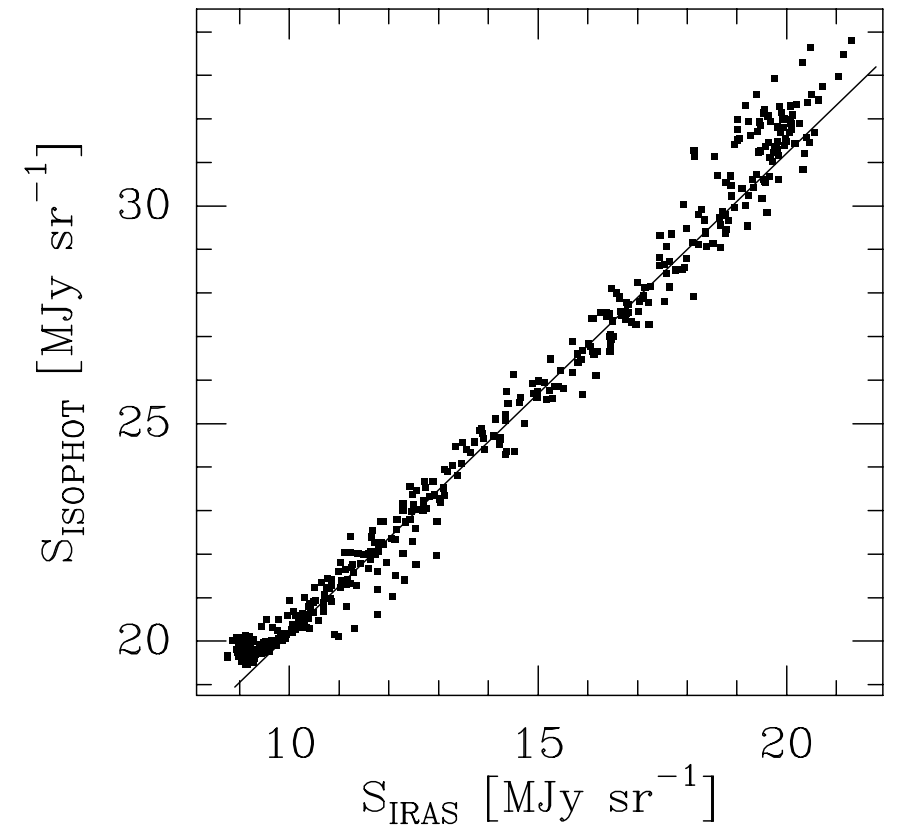

Fig. 1. The relation between the $100 \mu \mathrm{m}$ ISOPHOT and IRAS ISSA surface brightness values. The ISOPHOT time-ordered data have been tilted $5 \%$ in order to obtain lowest dispersion when compared with IRAS values (see text). The slope of the fitted line is 1.09. No zodiacal light has been subtracted from the ISOPHOT values.

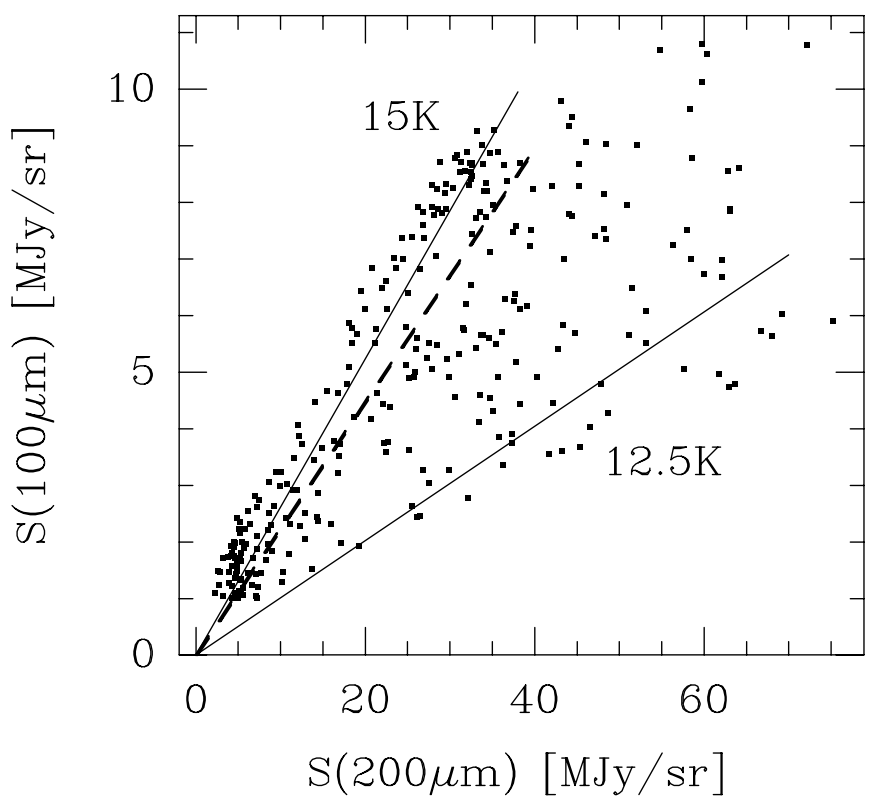

Fig. 2. Observed $100 \mu \mathrm{m}$ surface brightness as a function of the $200 \mu \mathrm{m}$ values. The diffuse background determined from regions with lowest surface brightness has been subtracted from the observed values and the $100 \mu \mathrm{m}$ data are convolved to the resolution of the $200 \mu \mathrm{m}$ observations. The solid lines correspond to dust colour temperatures $12.5 \mathrm{~K}$ and $15.0 \mathrm{~K}$. The dashed line shows the least squares line fitted to points $S(200 \mu \mathrm{m})<30 \mathrm{MJy} \mathrm{sr}^{-1}$.

IRAS ISSA surface brightness values is shown in Fig. 1. Because of the large temperature variations the relation 
Table 1. Summary of observed molecular emission lines. The columns are: (1) transition, (2) beam size, (3) spectral resolution (4) number of observed positions and (5) the average rms noise.

\begin{tabular}{rccrc}
\hline line & $\begin{array}{c}F W H M \\
\left({ }^{\prime \prime}\right)\end{array}$ & $\begin{array}{c}\Delta v \\
\left(\mathrm{~km} \mathrm{~s}^{-1}\right)\end{array}$ & positions & $\begin{array}{c}<\sigma_{\mathrm{RMS}}> \\
(\mathrm{K})\end{array}$ \\
\hline${ }^{12} \mathrm{CO}(2-1)$ & 23 & 0.054 & 83 & 0.17 \\
${ }^{13} \mathrm{CO}(1-0)$ & 46 & 0.114 & 83 & 0.069 \\
${ }^{13} \mathrm{CO}(2-1)$ & 24 & 0.057 & 532 & 0.14 \\
$\mathrm{C}^{18} \mathrm{O}(1-0)$ & 46 & 0.114 & 533 & 0.10 \\
$\mathrm{H}^{13} \mathrm{CO}+(1-0)$ & 57 & 0.144 & 174 & 0.040 \\
$\mathrm{DCO}+(2-1)$ & 35 & 0.087 & 176 & 0.061 \\
\hline
\end{tabular}

between $100 \mu \mathrm{m}$ and $200 \mu \mathrm{m}$ surfaces brightness values shows a significantly larger scatter (see Fig. 2). This is also decreased by the $5 \%$ adjustment of the $100 \mu \mathrm{m}$ responsivities.

The $100 \mu \mathrm{m}$ map was finally rescaled to the DIRBE surface brightness scale by multiplying the values with 0.85. The scaling was established by correlating DIRBE, IRAS and ISOPHOT surface brightness values in the area. Details of the procedure are given in Lehtinen et al. (in preparation). The $200 \mu \mathrm{m}$ observations were calibrated using the FCS measurements which were of good quality. The uncertainty in the absolute calibration of the $200 \mu \mathrm{m}$ map is expected to be below 20\% (see García-Lario 2000).

\subsection{Molecular line observations}

The molecular line observations were made with the SEST telescope during two session in February 1998 and February 1999. During the first observing period, the cloud was mapped in the lines ${ }^{12} \mathrm{CO}(2-1),{ }^{13} \mathrm{CO}(1-0)$ ${ }^{13} \mathrm{CO}(2-1), \mathrm{C}^{34} \mathrm{~S}(2-1)$ and $\mathrm{C}^{18} \mathrm{O}(1-0)$. Due to a problem with the receiver, the $\mathrm{C}^{18} \mathrm{O}(2-1)$ map remained uncompleted. In February 1999 observations were made in the $\mathrm{DCO}+(2-1)$ and $\mathrm{H}^{13} \mathrm{CO}+(1-0)$ lines.

During observations, the pointing was checked every two or three hours by observing bright $\mathrm{SiO}$ maser sources. The estimated pointing uncertainty is less than $5^{\prime \prime}$. Most observations were made in the frequency switching mode with a frequency throw $6 \mathrm{MHz}$. The chopper-wheel method was used for calibration and observed intensities are given in $T_{\mathrm{A}}^{*}$ units. The typical $\mathrm{rms}$ noise levels are given in Table 1.

The ${ }^{12} \mathrm{CO},{ }^{13} \mathrm{CO}$ and $\mathrm{C}^{18} \mathrm{O}$ maps cover practically the entire cloud L183 as seen in optical extinction. In the southern part, a fairly strong molecular line emission continues up to the edge of the map. The $\mathrm{H}^{13} \mathrm{CO}+(1-0)$ and $\mathrm{DCO}+(2-1)$ maps are smaller in size, but particularly in the case of $\mathrm{DCO}+$, they cover the whole region of significant emission. At the map boundaries, the $\mathrm{DCO}+$ intensity has dropped from a peak value $T_{\mathrm{A}} \approx 1.1 \mathrm{~K}$ to $\sim 0.25 \mathrm{~K}$ or below.

\subsection{Star counts}

The optical extinction in the L134 cloud complex was determined by means of star counts using a blue (IIaO + GG385 filter) and a red (IIIaF + RG630) ESO Schmidt plate (Plate Nos. 3713 and 3714) which were obtained on 16 April 1980. Star counts were performed using a reseau size of $2.8^{\prime} \times 2.8^{\prime}$. The total number of stars down to the limiting magnitude of each plate was counted (see Bok 1956 for the method).

In order to derive the extinction, the function $N(m)$ is needed, i.e. the number of stars per sq degree with magnitude $\leq m$ in a transparent comparison area close to the dark nebula. The tables of van Rhijn (1929) have often been used for this purpose (see Bok 1956). In the present case it was better to use the RGU photometric catalogue of Becker and Fenkart (1976) for Selected Area 107 which is located close $(l=5.7 \mathrm{deg}, b=41.3 \mathrm{deg})$ to the L134 complex. The $R$ and $G$ band star numbers for SA 107 were extracted from the compilation by Bahcall et al. (1985). These $N(m)$ curves were extrapolated beyond the limiting magnitudes $R=17.5$ and $G=18.5$, and were corrected to account for the somewhat different Galactic latitude ( $b=36 \mathrm{deg}$ ) of the L134 complex by using the tabulated results of Bahcall \& Soneira (1980) for their Galaxy model in the $B$ and $V$ bands.

An essential parameter of the $\log N(m)$ vs. $m$ calibration curve is its slope at the limiting magnitude of the star counts. In our case this slope turned out to be 0.22 at $G_{\mathrm{lim}}=20.1 \mathrm{mag}$ and $R_{\mathrm{lim}}=19.3 \mathrm{mag}$, respectively. We note that the $G$ and $R$ passbands of the Basle RGU photometric system with effective wavelengths of 463 and $638 \mathrm{~nm}$ are close enough to the wavelength ranges of our blue $(380-480 \mathrm{~nm})$ and red plate $(630-690 \mathrm{~nm})$.

The resulting extinction maps are shown in Fig. 3.

\section{Observational results}

\subsection{FIR maps}

The final far-infrared maps are shown in Fig. 4. The main structure seen in the far-infrared maps (Fig. 4a-b) is a wedge pointing northwards. The intensity is highest close to the head of the wedge, and drops rapidly towards the north and more slowly towards the south. There are clear differences in the distributions at $100 \mu \mathrm{m}$ and $200 \mu \mathrm{m}$. There are two broad $200 \mu \mathrm{m}$ maxima, one close to the optical extinction peak and previously known mm- and sub-mm continuum source (Ward-Thompson et al. 1994) and another $\sim 5^{\prime}$ towards SE. In the $100 \mu \mathrm{m}$ map only the latter peak is visible. The relative strength of the $200 \mu \mathrm{m}$ emission is larger in the north, west and southwest while the $100 \mu \mathrm{m}$ emission is stronger in the southeast. In the west the $100 \mu \mathrm{m}$ emission seems to extend further out, although the surface brightness is already close to the background level.

The $200 \mu \mathrm{m}$ emission is caused entirely by the large classical grains and at $100 \mu \mathrm{m}$ the contribution from small grains is expected to be small, $\lesssim 10 \%$. Dust temperature 


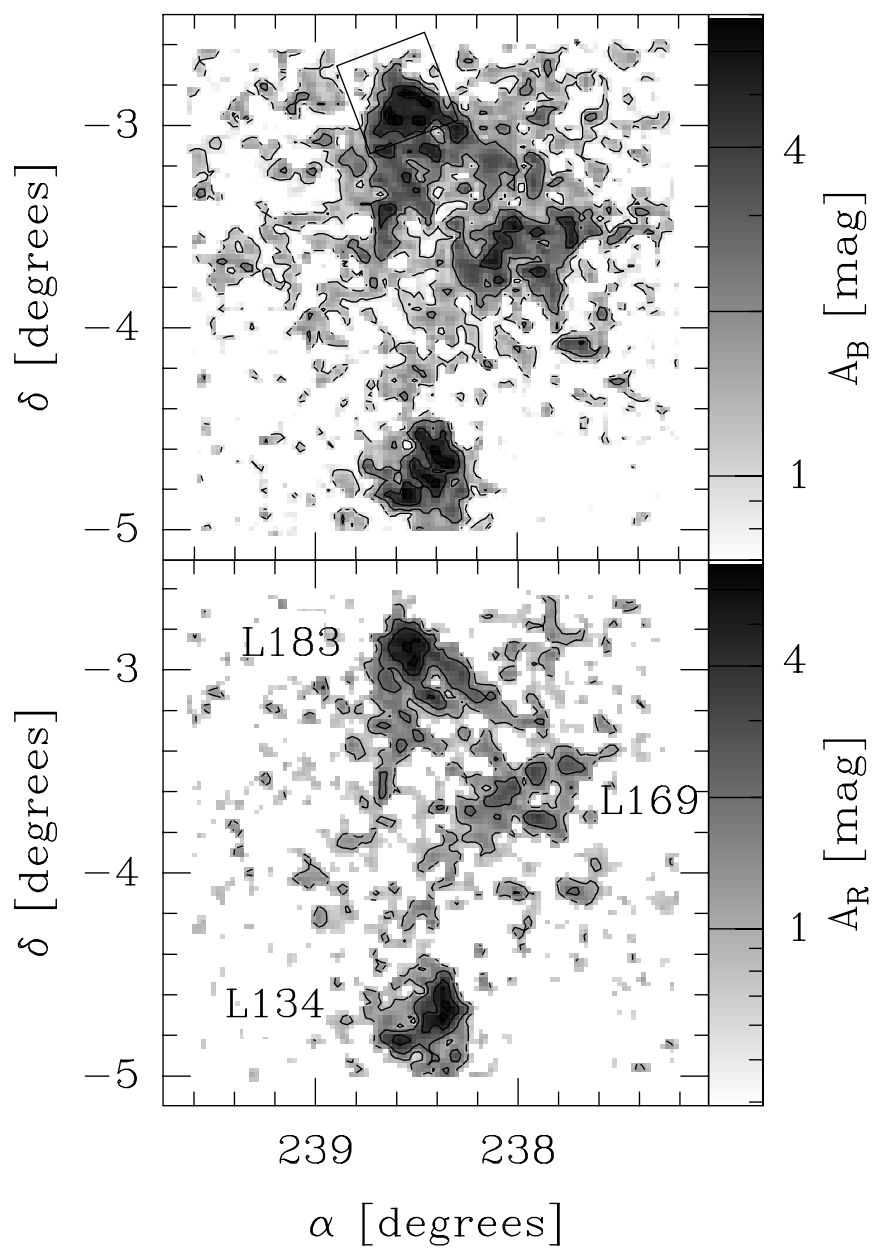

Fig. 3. The extinction maps of the L134 dark cloud complex derived from starcounts in the $R$ - (lower frame) and $B$-bands (upper frame). The area mapped with ISOPHOT is indicated with a square. Individual clouds L134, L183 and L169 are indicated in the figure. The contour levels are at 1,2 and $4^{\mathrm{m}}$.

map was derived assuming that large grains emit according to a modified black body radiation $\nu^{2} B\left(\nu, T_{\mathrm{d}}\right)$. The background corresponding to the lowest surface brightness values within the mapped area was first subtracted. Average $100 \mu \mathrm{m}$ surface brightness values were calculated corresponding to each $200 \mu \mathrm{m}$ measurement. Values were colour corrected and the dust temperature was obtained by the fitting of the modified Planck curve. Since the colour correction depends weakly on the assumed temperature the procedure was repeated once using the temperature derived from the initial fit.

The dust temperature in Fig. 4c has a well defined minimum close to the position of the continuum source. The minimum temperature in the $200 \mu \mathrm{m}$ core is below $\sim 12 \mathrm{~K}$ while in the north and west the temperature rises rapidly to $\sim 16 \mathrm{~K}$. There is no temperature maximum at the $100 \mu \mathrm{m}$ emission maximum and the position does not differ in any way from its surroundings. The $5 \%$ tilting of the $100 \mu \mathrm{m}$ map (Sect. 2.1) changed the temperatures by up to $\sim 1 \mathrm{~K}$ at the eastern and western borders (the first and last scan lines in the map). As the average responsivity was not changed the temperature in the centre, i.e. in the cold core, was not affected.

The correlation between $100 \mu \mathrm{m}$ and $200 \mu \mathrm{m}$ surface brightness values (Fig. 2) shows considerable scatter. The scatter was somewhat reduced by the correction applied to the relative responsivity of the $100 \mu \mathrm{m}$ FCS measurements (see Sect. 2.1). A further modification of the responsivities would have very little effect on the scatter, which is caused by true variations of the dust properties. The variations are reflected in the dust temperature distribution.

A linear least squares fit to the data points in Fig. 2 gives a relation $S_{\text {fit }}(200 \mu \mathrm{m})=(0.14 \pm 0.01) S(100 \mu \mathrm{m})+$ $(1.30 \pm 0.06)$. The fitting procedure takes into account the uncertainty in both variables. The statistical error of the intercept in particular is small compared with the uncertainty due to calibration and the background subtraction. Data at positions where the background subtracted values of $S(200 \mu \mathrm{m})$ were below $30 \mathrm{MJy} \mathrm{sr}^{-1}$ (i.e. outside the cloud core $)$ give a relation $S_{\text {fit }}(200 \mu \mathrm{m})=(0.22 \pm 0.01)$ $S(100 \mu \mathrm{m})$. The limit of $30 \mathrm{MJysr}^{-1}$ at $200 \mu \mathrm{m}$ corresponds approximately to the $50 \mathrm{MJy} \mathrm{sr}^{-1}$ contour in Fig. 4 where no background subtraction was made. According to Fig. 3 the excluded area corresponds also to the region with highest extinction, $A_{\mathrm{B}} \gtrsim 4^{\mathrm{m}}$. Based on the derived dependence we calculate $\Delta S(200 \mu \mathrm{m})=S_{\text {obs }}(200 \mu \mathrm{m})$ $S_{\text {fit }}(200 \mu \mathrm{m})$, which is the difference of the observed $200 \mu \mathrm{m}$ surface brightness and the predicted value based on the $100 \mu \mathrm{m}$ data. The resulting map of $\Delta S(200 \mu \mathrm{m})$ (Fig. 5) shows the $200 \mu \mathrm{m}$ excess that can be due to either cold dust or dust with enhanced emissivity at the longer wavelength. The $200 \mu \mathrm{m}$ optical depth was calculated from the observed intensities as $\tau(200 \mu \mathrm{m})=S / B(T)$. Here $B(T)$ is the black body intensity at temperature $T$ as read from Fig. 4c. The resulting map, which shows the column density distribution of large dust grains, is rather similar to the $200 \mu \mathrm{m}$ intensity distribution. Due to its low colour temperature, however, the main core is much more pronounced. The region of the $100 \mu \mathrm{m}$ peak is correspondingly weaker and is visible only as an extension of the main core. Figure 6 shows the $200 \mu \mathrm{m}$ surface brightness in relation to the $200 \mu \mathrm{m}$ optical depth. While the SE core is also visible as a separate intensity peak in the $200 \mu \mathrm{m}$ map, the large grains are clearly concentrated in the NW core. A similar relation is seen between the $200 \mu \mathrm{m}$ emission and the dust colour temperature. In the temperature map the SE peak is completely invisible.

The cloud mass was calculated from the $200 \mu \mathrm{m}$ optical depth using the value $\sigma_{\mathrm{H}}=2.5 \times 10^{-25} \mathrm{~cm}^{2}$ for the effective dust cross section per hydrogen atom at $200 \mu \mathrm{m}$ (Lehtinen et al. 1998). The mass within $10^{\prime}$ of position $(0,0)$ is $25 M_{\odot}$ for $d=100 \mathrm{pc}$. Alternatively, the mass contained within the contour $\tau(200 \mu \mathrm{m})=6 \times 10^{-4}$ is $28 M_{\odot}$. The main uncertainty is in the value of $\sigma_{\mathrm{H}}$ and use of the formula given by Boulanger et al. (1996), $\sigma_{\mathrm{H}}=10^{-25}(\lambda / 250 \mu \mathrm{m})^{-2} \mathrm{~cm}^{-2}$, would result in some $50 \%$ higher mass estimates. We note that the latter $\sigma_{\mathrm{H}}$ value is for diffuse medium, while the Lehtinen et al. value is for a dense globule similar to L183. 


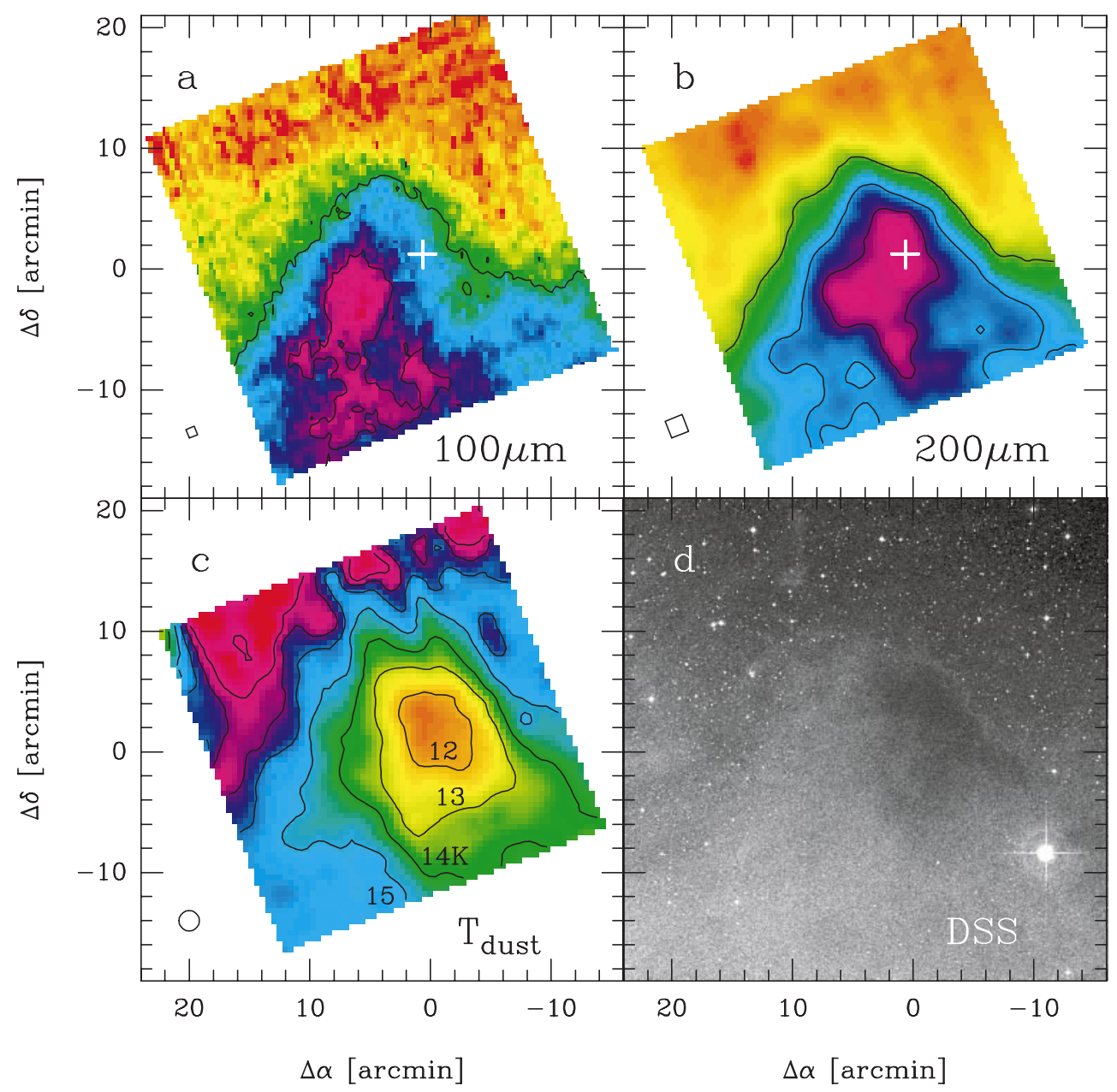

Fig. 4. a)-b) The $100 \mu \mathrm{m}$ and $200 \mu \mathrm{m}$ ISOPHOT maps of the cloud L183. The cross indicates the position of the continuum source detected by Ward-Thompson et al. (1994). The $(0,0)$ position is RA $=15^{\mathrm{h}} 51^{\mathrm{m}} 30^{\mathrm{s}}$ DEC $=-2^{\circ} 43^{\prime} 31^{\prime \prime}$. In the case of $100 \mu \mathrm{m}$ the contours are at 15 and $20 \mathrm{MJy} \mathrm{sr}^{-1}$ and for $200 \mu \mathrm{m}$ the contour levels are 30, 50 and $70 \mathrm{MJy} \mathrm{sr}^{-1}$. The small squares at the lower left corner indicate the pixel sizes, $44^{\prime \prime}$ for $100 \mu \mathrm{m}$ and $89^{\prime \prime}$ for the $200 \mu \mathrm{m}$ map. c) The map of dust colour temperature calculated from the $100 \mu \mathrm{m}$ and $200 \mu \mathrm{m}$ ISOPHOT observations. The contours are at intervals of $1 \mathrm{~K}$ starting with $12 \mathrm{~K}$. The effective resolution is $100^{\prime \prime}$. d) Blue image from the Digitized Sky Survey.

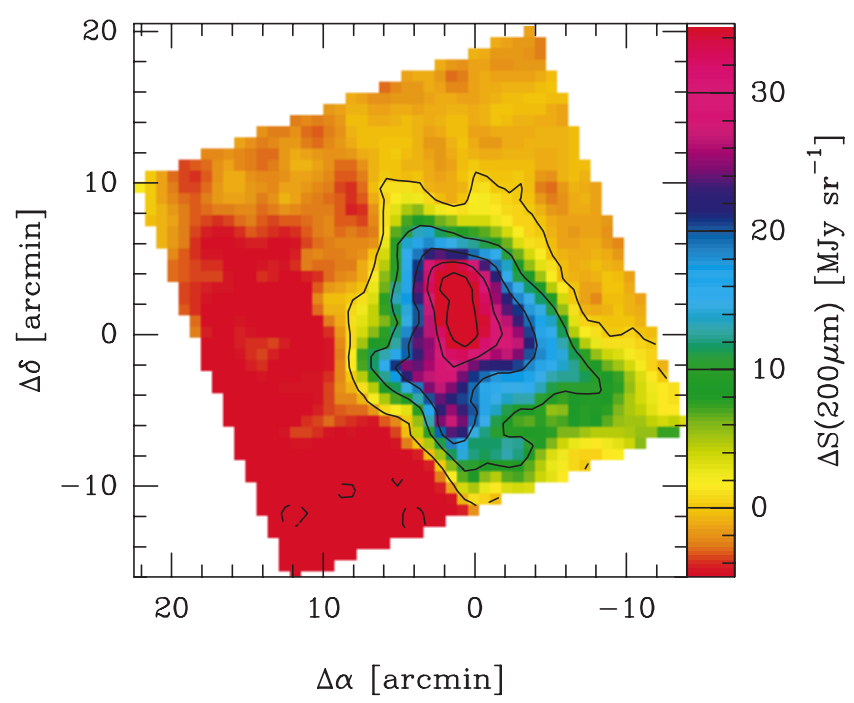

Fig. 5. Distribution of $\Delta S(200 \mu \mathrm{m}) . \Delta S(200 \mu \mathrm{m})$ is the difference between the observed $200 \mu \mathrm{m}$ surface brightness and the least squares prediction calculated from the $100 \mu \mathrm{m}$ values.

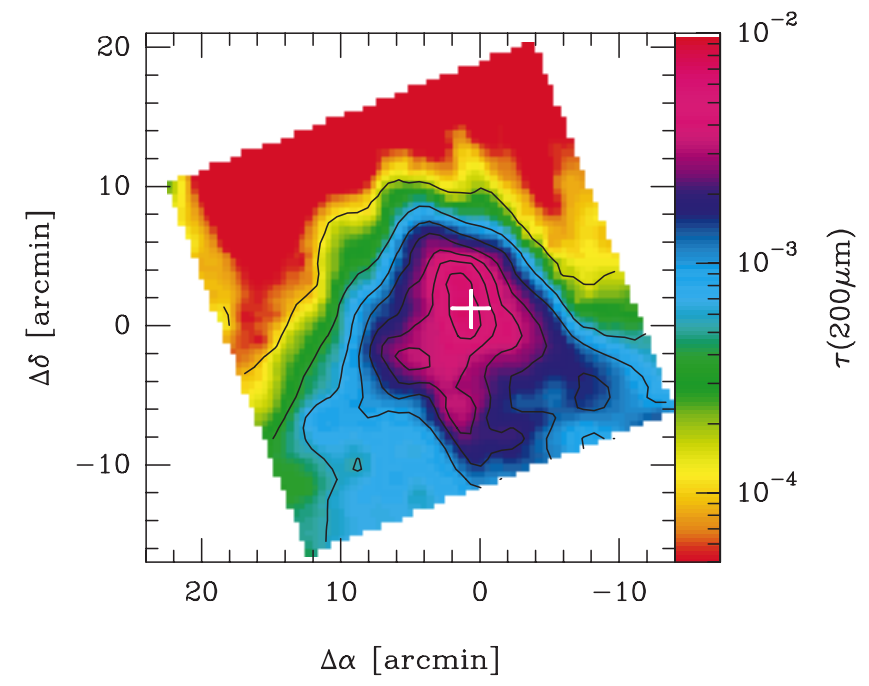

Fig. 6. $200 \mu \mathrm{m}$ surface brightness (contours) and the calculated optical depths at the same wavelength. The contours start at $15 \mathrm{MJy} \mathrm{sr}^{-1}$ and are drawn at intervals of $15 \mathrm{MJy} \mathrm{sr}^{-1}$. 
As shown in Fig. 5, there is a clear $200 \mu \mathrm{m}$ excess in the centre of L183. This could be due to a decrease in the physical temperature of the dust grains induced by the decreasing intensity of the radiation field, or by increased overall FIR emissivity of the grains. Another possibility would be a change in grain properties, i.e. increased emissivity at the longer wavelength, leading to a change of the dust emissivity index. Both explanations are related to the increasing density in the cloud centre. Both theoretical studies (e.g. Ossenkopf 1993; Wright 1987) and recent PRONAOS observations (Bernard et al. 1999; Stepnik et al. 2001) indicate that dust emission properties are likely to change in dense and cold clouds. This could be the result of grain growth, or possibly the formation of grain aggregates. In a forthcoming paper (Juvela et al., in preparation) we will study the far-infrared dust emission using radiative transfer models. Further discussion about the dust temperatures and changes in the dust emissivity law will be deferred to that paper.

\subsection{Distribution of molecular line emission}

Figure 7 shows the distributions of the integrated antenna temperature in lines ${ }^{12} \mathrm{CO}(2-1),{ }^{13} \mathrm{CO}(2-1), \mathrm{C}^{18} \mathrm{O}(1-0)$, $\mathrm{H}^{13} \mathrm{CO}+(1-0)$ and $\mathrm{DCO}+(2-1)$.

The CO lines follow the general morphology seen in the maps by Snell (1981) and Swade et al. (1989a). The ${ }^{12} \mathrm{CO}$ emission increases towards the south while $\mathrm{C}^{18} \mathrm{O}$ and ${ }^{13} \mathrm{CO}(1-0)$ delineate the dense core close to the given centre position. The ${ }^{13} \mathrm{CO}(1-0)$ emission extends towards the southwest, however, where the ${ }^{13} \mathrm{CO}(2-1)$ emission peaks well outside the core seen in $\mathrm{C}^{18} \mathrm{O}$. The distributions of $\mathrm{H}^{13} \mathrm{CO}+(1-0)$ and $\mathrm{DCO}+(2-1)$ are clearly different from the $\mathrm{CO}$ species. The distribution of $\mathrm{H}^{13} \mathrm{CO}+(1-0)$ is similar to what was seen by Guélin et al. (1982). However, due to the better resolution of the present study the emission area is better resolved and the map is more structured. There is a second emission peak south of the centre at position $(1,-4)$. The morphology of the $\mathrm{DCO}+(2-1)$ emission follows closely that of the $\mathrm{H}^{13} \mathrm{CO}+(1-0)$. The emission is concentrated along a narrow, but clearly resolved ridge with some extension west of the centre position. The western extension is seen in both lines, although it was not visible in the Guélin et al. $\mathrm{H}^{13} \mathrm{CO}$ map. In the centre, the $\mathrm{DCO}+$ emission seems to be more concentrated around the peak position. However, this is mainly due to the smaller beam size of the $\mathrm{DCO}+$ observations.

Maps of $\mathrm{C}^{18} \mathrm{O}(1-0),{ }^{13} \mathrm{CO}(1-0)$ and ${ }^{13} \mathrm{CO}(2-1)$ emission in different velocity intervals are shown Figs. 8-10. In the lowest radial velocity bin, $\mathrm{C}^{18} \mathrm{O}$ emission is seen both northeast of the position $(0,0)$ and towards the western edge of the map. In the interval $2.0-2.5 \mathrm{~km} \mathrm{~s}^{-1}$, strong emission still exists in the western part of the map. The main core is visible in all three higher velocity intervals. Below $3 \mathrm{~km} \mathrm{~s}^{-1}$ the emission extends between the positions of the $100 \mu \mathrm{m}$ and $200 \mu \mathrm{m}$ maxima (see Fig. 4) while at the highest radial velocities the emission is concentrated
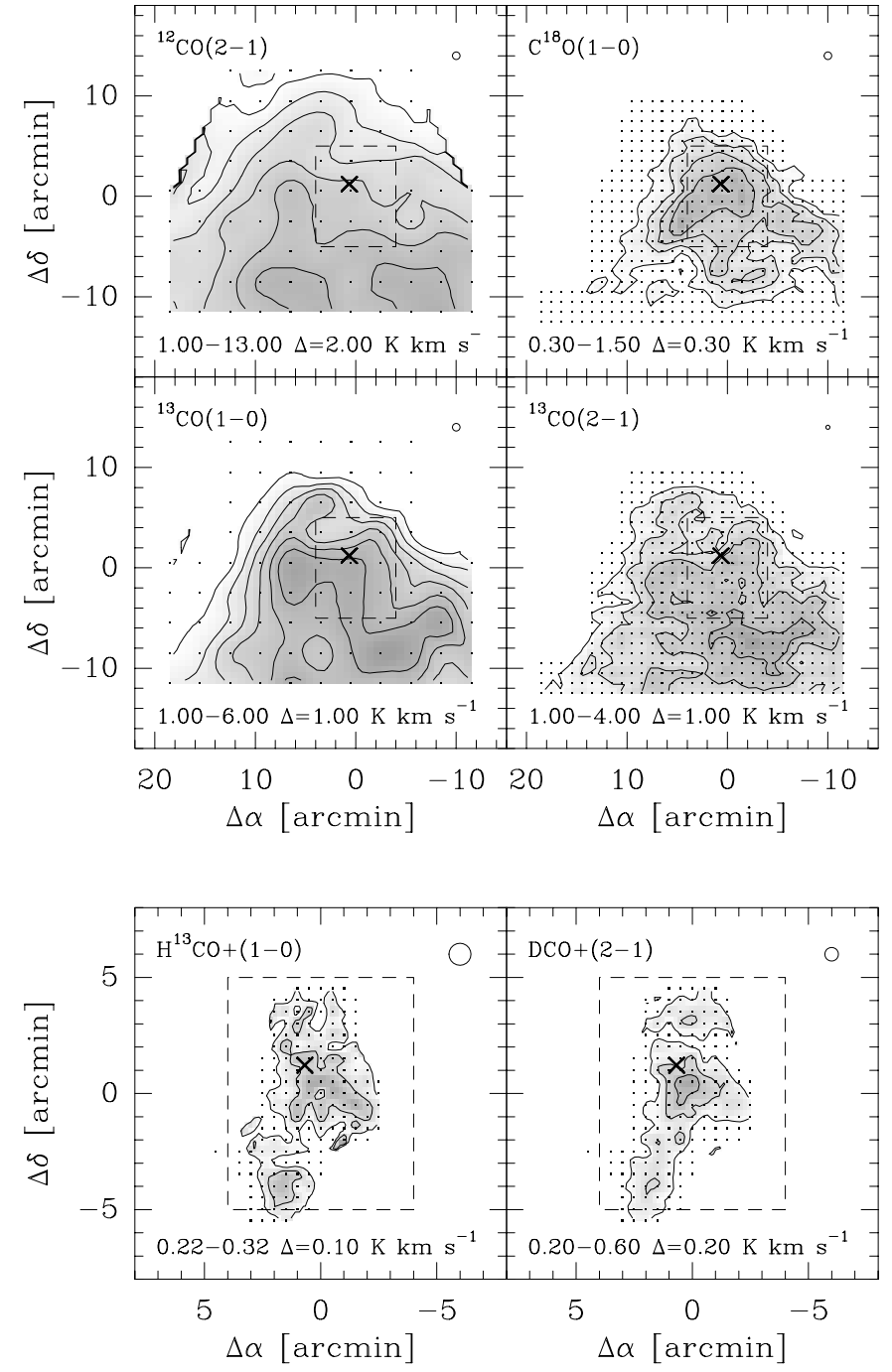

Fig. 7. Maps of the integrated line areas: ${ }^{12} \mathrm{CO}(1-0), \mathrm{C}^{18}(1-0)$, ${ }^{13} \mathrm{CO}(1-0),{ }^{13} \mathrm{CO}(2-1), \mathrm{H}^{13} \mathrm{CO}+(1-0)$ and $\mathrm{DCO}+(2-1)$. The dashed lines in each frame indicate the location of the $\mathrm{DCO}+$ core.

around position $(0,0)$ only. The same general morphology and dependence on the radial velocity is repeated in the ${ }^{13} \mathrm{CO}$ maps. The relative intensity of the ${ }^{13} \mathrm{CO}$ lines is, however, higher in the south. As the radial velocity increases the emission maximum moves over the $\mathrm{C}^{18} \mathrm{O}$ core from northeast to southwest.

The $\mathrm{DCO}+(2-1)$ and $\mathrm{H}^{13} \mathrm{CO}+(2-1)$ in Figs. 11-12 show similar behaviour. At low velocity, the emission is concentrated in the south around position $(2,-3)$ while at other velocities the maximum is reached close to position $(0,0)$. There are also some differences, and $\mathrm{H}^{13} \mathrm{CO}+$ is relatively stronger around the emission peak in the south.

\section{Models of radiative transfer and column densities of molecular gas}

LTE column density estimates were computed based on the ${ }^{13} \mathrm{CO}(1-0)$ and $\mathrm{C}^{18} \mathrm{O}(1-0)$ observations. The derived 


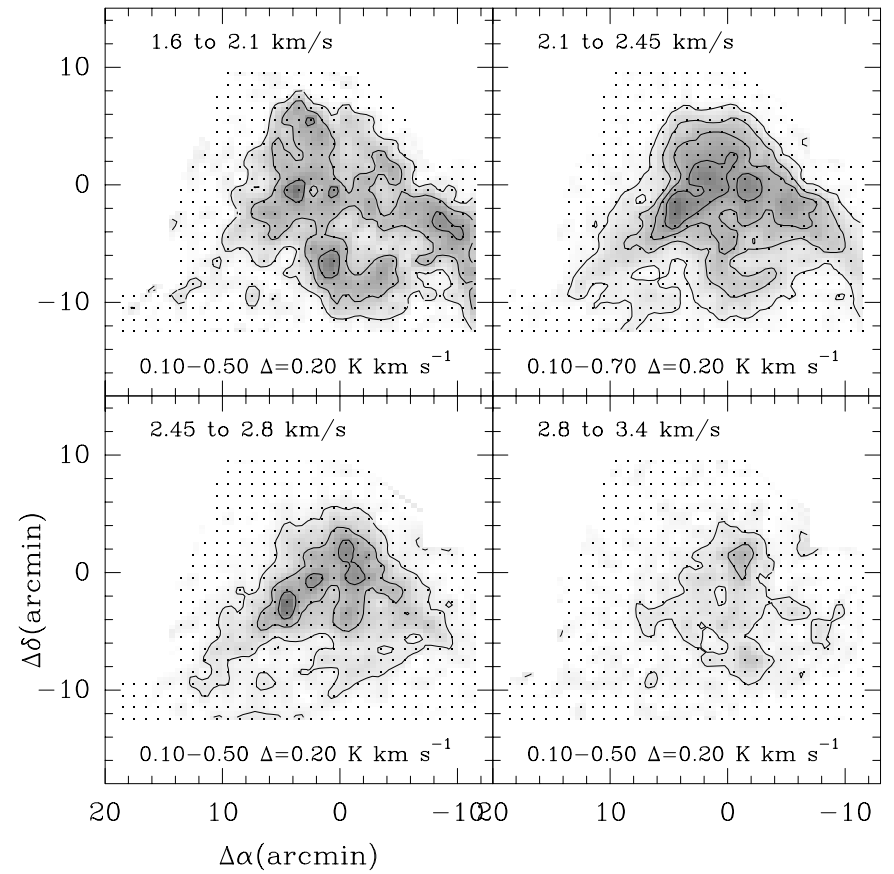

Fig. 8. $\mathrm{C}^{18} \mathrm{O}(1-0)$ integrated antenna temperature in four velocity intervals. The velocity intervals are given in the upper part of the panels. The range of contour levels and the step between consecutive contours are written at the bottom of the panels.

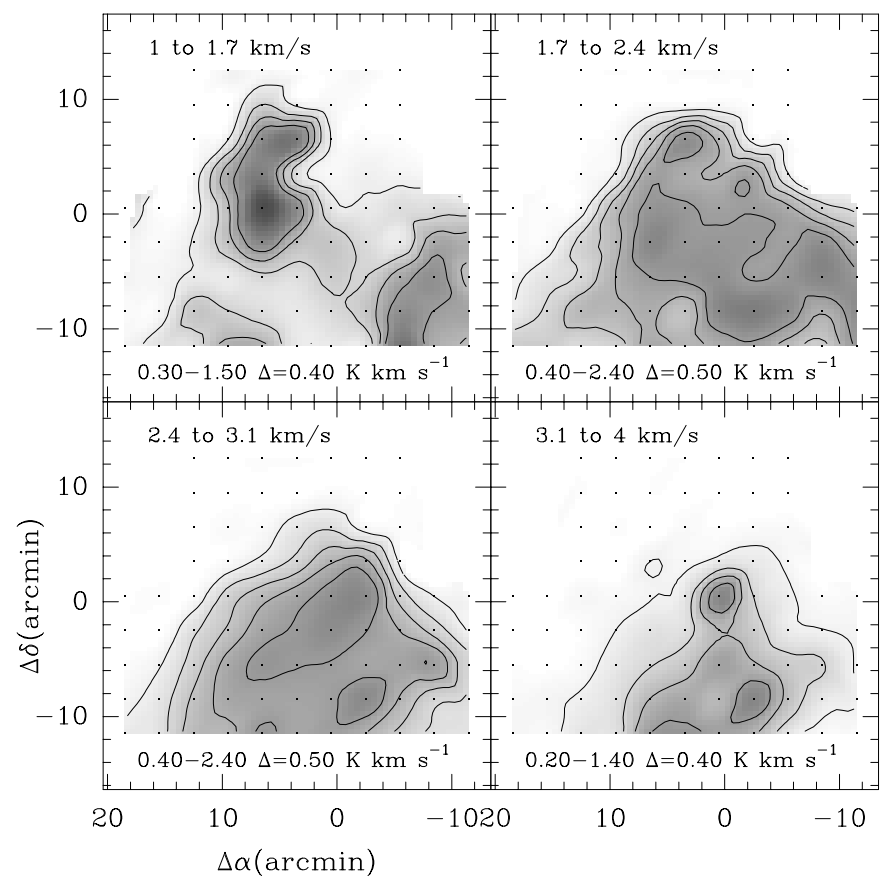

Fig. 9. ${ }^{13} \mathrm{CO}(1-0)$ integrated antenna temperature in four velocity intervals. The velocity intervals and the contour values are indicated in the panels.

excitation temperatures are between 7 and $9 \mathrm{~K}$. Compared with the rest of the cloud, the excitation temperature seems to be systematically $1 \mathrm{~K}$ lower in the northern part close to the column density maximum. However, the difference is small and we derive column densities assuming a

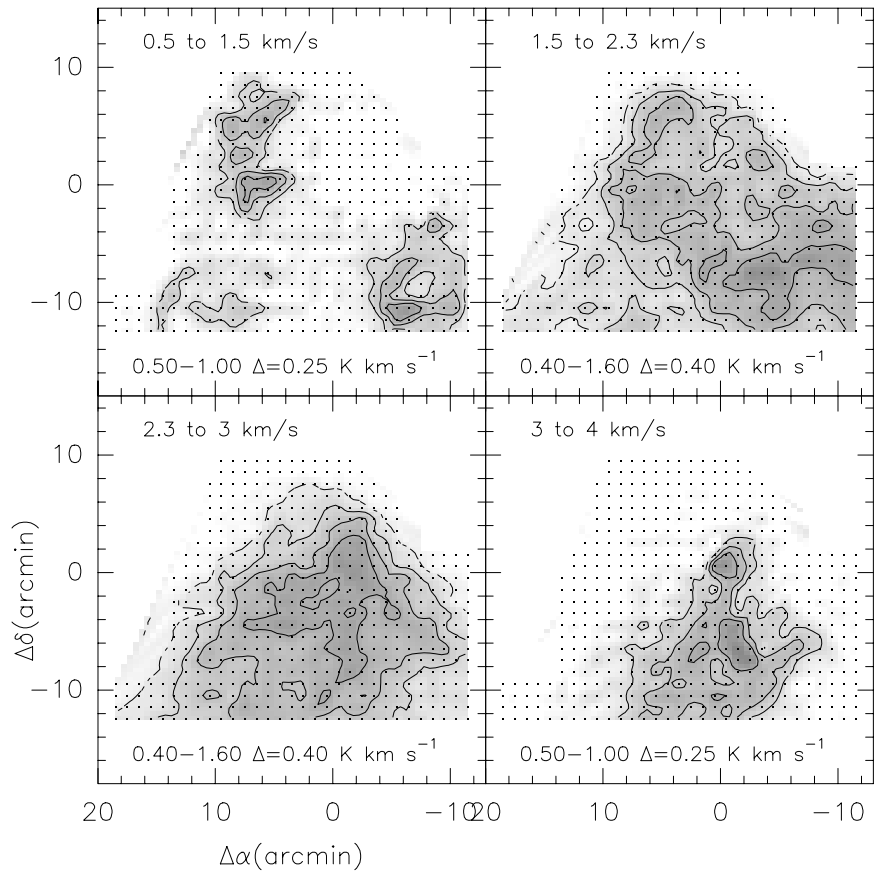

Fig. 10. ${ }^{13} \mathrm{CO}(2-1)$ integrated antenna temperature in four velocity intervals. The velocity intervals and the contour values are indicated in the panels.

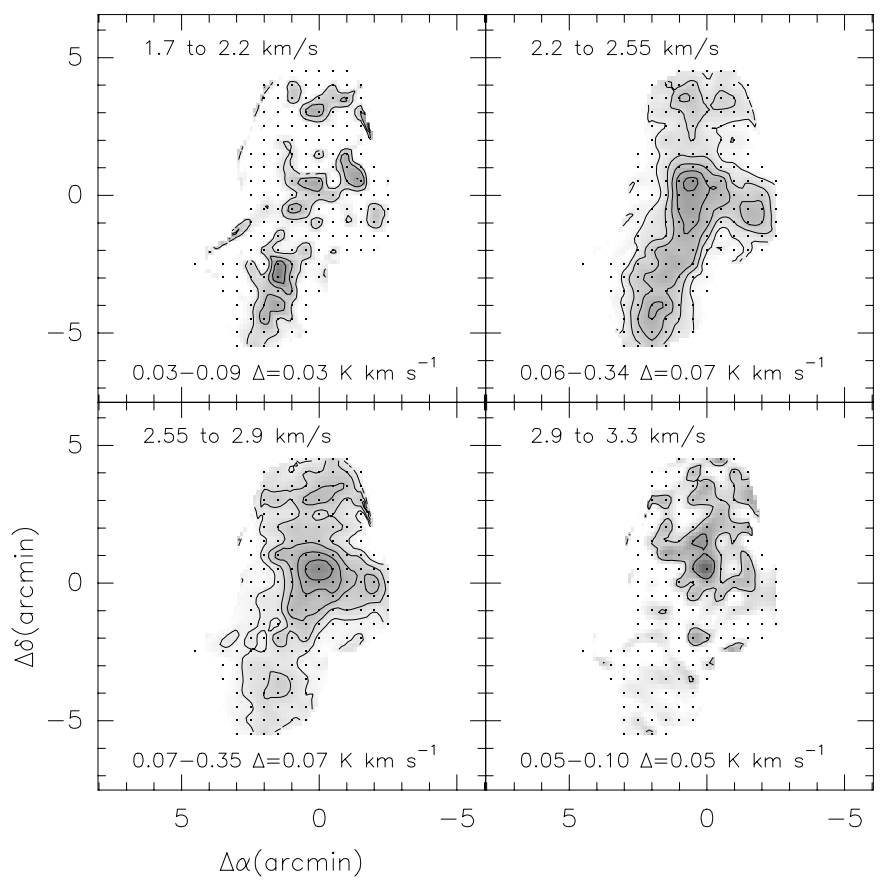

Fig. 11. DCO $+(2-1)$ integrated antenna temperature. The velocity intervals and the contour values are indicated in the panels.

constant excitation temperature of $T_{\text {ex }}=8.4 \mathrm{~K}$. The LTE column densities were calculated according to the formula given by Harjunpää et al. (1996) and are shown in Fig. 13. In the conversion from $\mathrm{C}^{18} \mathrm{O}$ to $\mathrm{H}_{2}$ column densities, a fractional $\mathrm{C}^{18} \mathrm{O}$ abundance of $1 \times 10^{-7}$ was assumed, corresponding to the quiescent cloud positions in Harjunpää et al. (1996) and Frerking et al. (1982). 


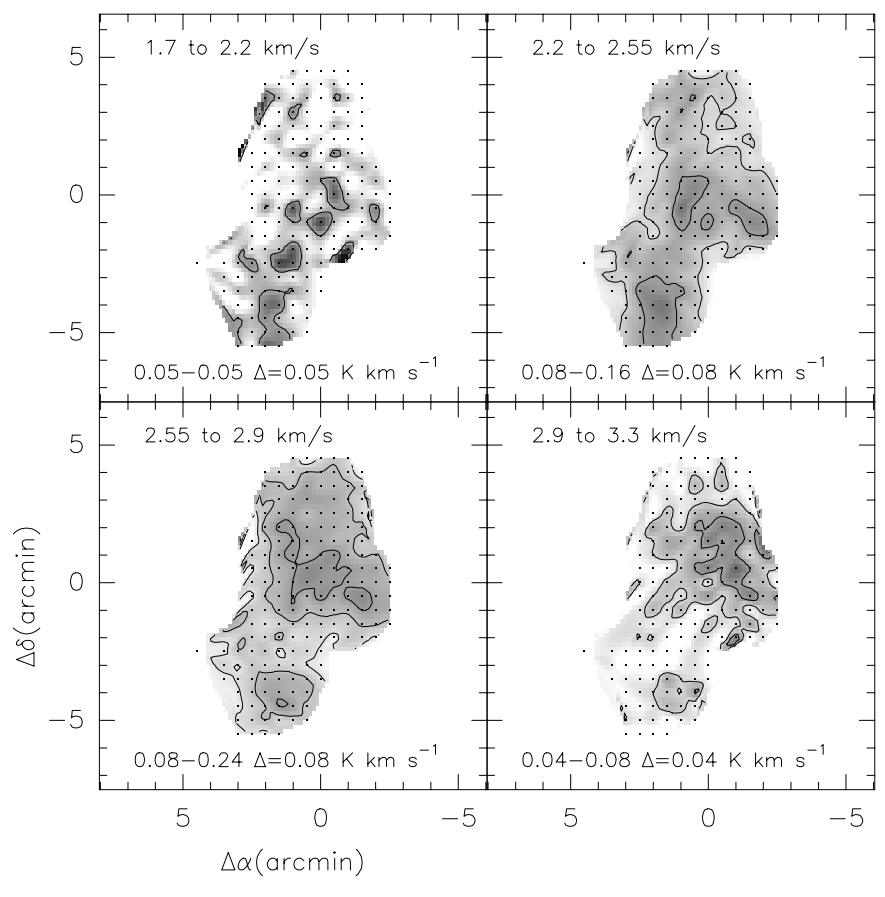

Fig. 12. $\mathrm{H}^{13} \mathrm{CO}+(1-0)$ integrated antenna temperature. The velocity intervals and the contour values are indicated in the panels.

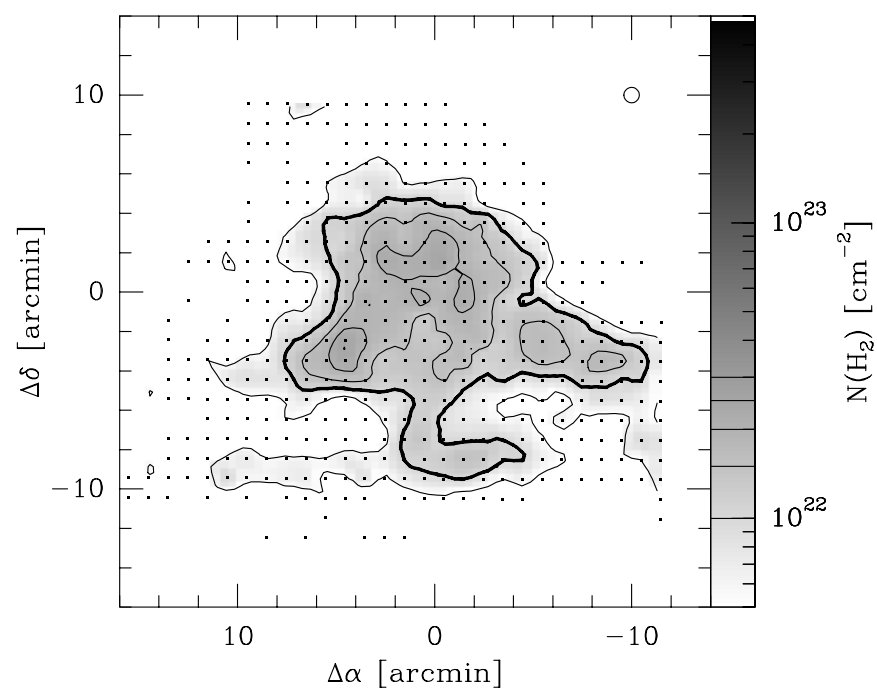

Fig. 13. LTE hydrogen column density as derived from the ${ }^{13} \mathrm{CO}(1-0)$ and $\mathrm{C}^{18} \mathrm{O}(1-0)$ observations. The contours start at value $N\left(\mathrm{H}_{2}\right)=5 \times 10^{21} \mathrm{~cm}^{-2}$ and are drawn at intervals of $5 \times$ $10^{21} \mathrm{~cm}^{-2}$ (the thick contour line denotes the value $N\left(\mathrm{H}_{2}\right)=$ $\left.1 \times 10^{22} \mathrm{~cm}^{-2}\right)$.

From the derived column densities we obtain a value of $\sim 47 M_{\odot}$ for the cloud mass within $10^{\prime}$ of the centre position, or some $35 M_{\odot}$ for the mass contained within the contour $N\left(\mathrm{H}_{2}\right)=1.0 \times 10^{22} \mathrm{~cm}^{-2}$.

For comparison, the $\mathrm{C}^{18} \mathrm{O}$ and ${ }^{13} \mathrm{CO}$ emission were modelled with spherically-symmetric model clouds where the radiative transfer problem was solved with Monte Carlo methods (see Juvela et al. 1997). While spherical symmetry presents an extreme simplification of true density distribution, the models will still be more realistic than the assumptions underlying the LTE calculations (see e.g. Padoan et al. 2000). The model clouds are assumed to be isothermal with radial density dependence of $n \sim r^{-1.5}$ and with density contrast 20 between cloud centre and outer boundary. The free parameters of the models were the cloud size, central density and the kinetic temperature. The $\mathrm{C}^{18} \mathrm{O}(1-0),{ }^{13} \mathrm{CO}(1-0)$ and ${ }^{13} \mathrm{CO}(2-1)$ spectra within $10^{\prime}$ radius of the position $(0,0)$ were compared with spectra calculated from the radiative transfer model and the free parameters were adjusted in order to obtain the best fit. The gas distribution is asymmetric with respect to the centre position and the ${ }^{13} \mathrm{CO}$ emission is displaced with respect to $\mathrm{C}^{18} \mathrm{O}$. Therefore, the results can not be expected to be very accurate. However, although the listed free parameters are not well constrained, the column density estimates should be more reliable.

Assuming a constant kinetic temperature of $10 \mathrm{~K}$, the best fit was obtained with a model having a mass of $40 M_{\odot}$. The mass estimate is not very sensitive to the assumed kinetic temperature. A similar model with $T_{\text {kin }}$ increasing linearly from $8.0 \mathrm{~K}$ in the centre to $15 \mathrm{~K}$ on the cloud surface resulted in a mass estimate of $34 M_{\odot}$. The quality of the fit was, however, marginally better in the isothermal model.

The spectral lines do not show signs of cloud collapse. Several ${ }^{13} \mathrm{CO}$ spectra have double peaked, asymmetric profiles but comparison with optically thinner $\mathrm{C}^{18} \mathrm{O}$ indicates that these are caused by separate emission components rather than self-absorption. Gaussian fits give average linewidths of $\sim 0.7 \mathrm{~km} \mathrm{~s}^{-1}$ for both ${ }^{13} \mathrm{CO}(2-1)$ and $\mathrm{C}^{18} \mathrm{O}(1-0)$. The virial mass was estimated according to equation

$M_{\mathrm{vir}}=\left(\frac{\sigma_{3 \mathrm{D}}}{C}\right)^{2} R$

where $\sigma_{3 \mathrm{D}}$ is the three-dimensional velocity dispersion, $R$ the radius of the cloud and $C$ is a constant depending on the assumed model of the cloud. The value of $C$ is $0.0508 \mathrm{~km} \mathrm{~s}^{-1} \sqrt{\mathrm{pc} / M_{\odot}}$ for a homogeneous sphere and $0.0463 \mathrm{~km} \mathrm{~s}^{-1} \sqrt{\mathrm{pc} / M_{\odot}}$ for density distribution $n \sim r^{-1}$ (see Liljeström 1991). Assuming gas temperature $13 \mathrm{~K}$ and $R \sim 10^{\prime}$ the observed velocity dispersion corresponds to virial masses of $54 M_{\odot}$ and $44 M_{\odot}$ for the two models. In the presence of noise the $F W H M$ derived from Gaussian fits may, however, be biased and the average $F W H M$ read directly from the spectra is smaller, $\sim 0.5 \mathrm{~km} \mathrm{~s}^{-1}$, lowering the virial mass estimates to $36 M_{\odot}$ and $30 M_{\odot}$, correspondingly. The previous analysis ignores both the external pressure and the presence of velocity gradients. The cloud is, however, approximately in virial equilibrium. Even though the cloud as a whole may not be collapsing it can still contain collapsing fragments. In fact, there is evidence of several pre-protostellar cores in L183 (Lehtinen et al. 2000).

We will return to the modelling of the molecular line data in a future paper (Juvela et al., in preparation) where 


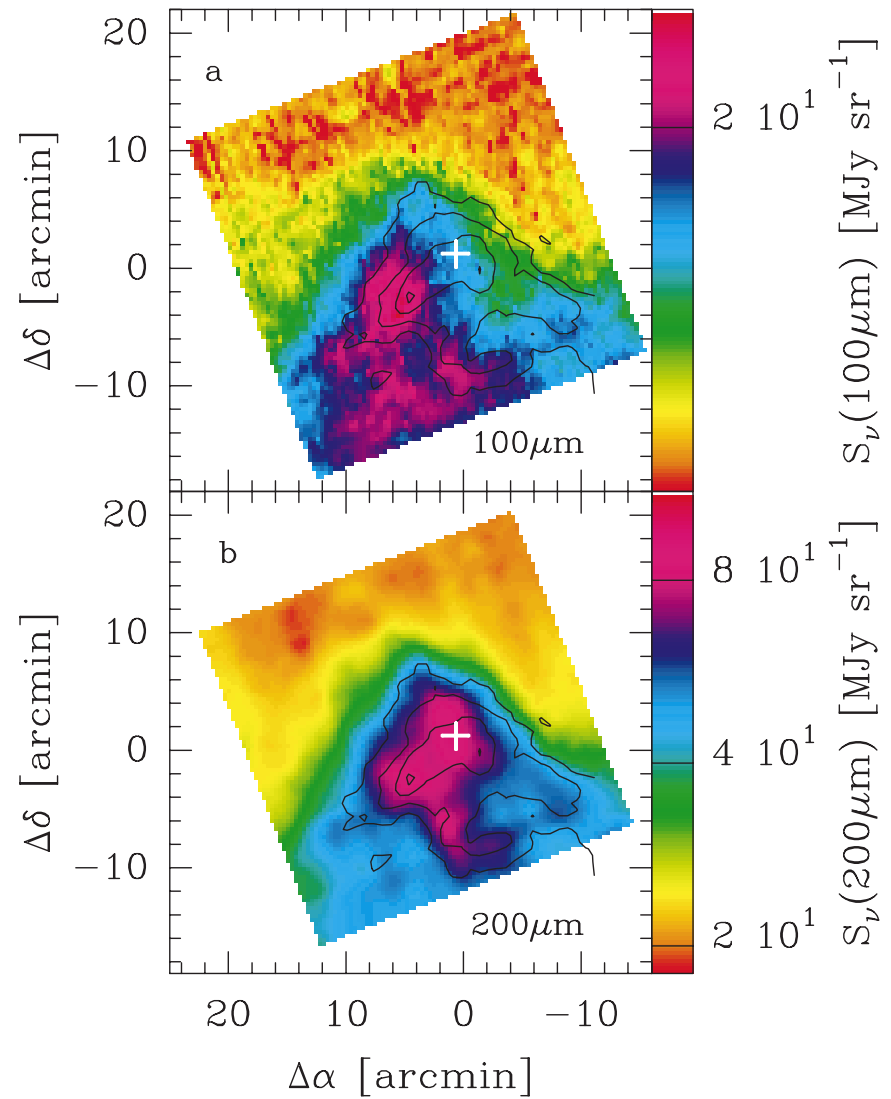

Fig. 14. The far-infrared maps of L183 at $100 \mu \mathrm{m}$ and $200 \mu \mathrm{m}$ with $\mathrm{C}^{18} \mathrm{O}(1-0)$ contours overlaid. The contours are drawn between $0.4 \mathrm{~K} \mathrm{~km} \mathrm{~s}^{-1}$ and $1.6 \mathrm{~K} \mathrm{~km} \mathrm{~s}^{-1}$ at intervals of $0.3 \mathrm{~K} \mathrm{~km} \mathrm{~s}^{-1}$.

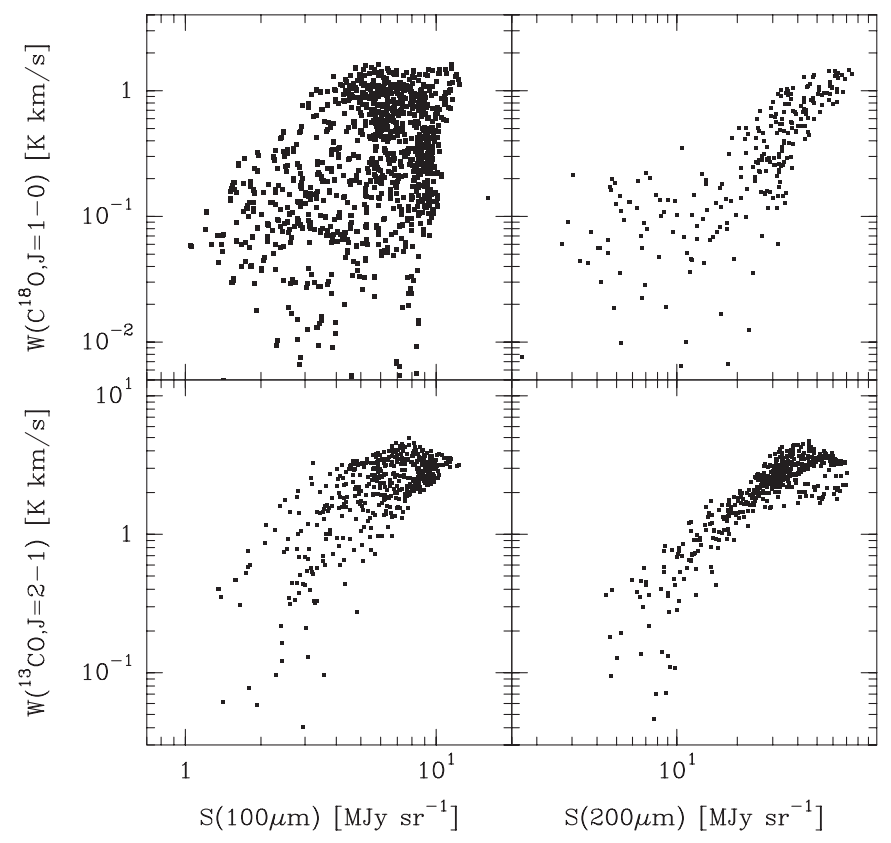

Fig. 15. $\mathrm{C}^{18} \mathrm{O}(1-0)$ and ${ }^{13} \mathrm{CO}(2-1)$ line areas as the function of $100 \mu \mathrm{m}$ and $200 \mu \mathrm{m}$ surface brightness. In plots containing the $200 \mu \mathrm{m}$ surface brightness values the original data are smoothed to resolution of $100^{\prime \prime}$. In the case of $100 \mu \mathrm{m}$ the corresponding resolution is $70^{\prime \prime}$.

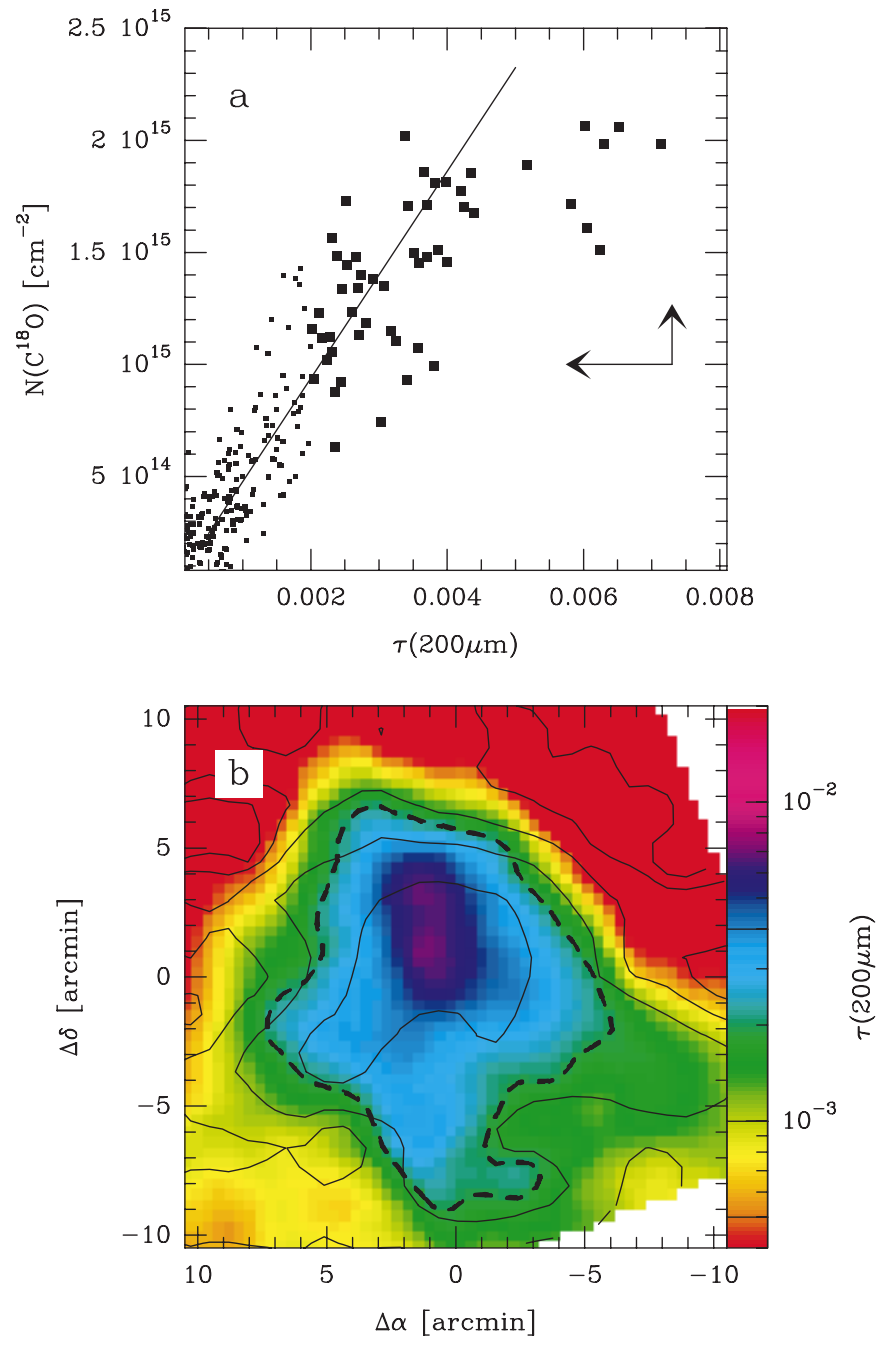

Fig. 16. a) Correlation between the dust optical depth at $200 \mu \mathrm{m}$ and the $\mathrm{C}^{18} \mathrm{O}$ column density derived from $\mathrm{C}^{18} \mathrm{O}(1-0)$ observations, assuming a constant excitation temperature of $8.4 \mathrm{~K}$. The arrows indicate the shift if $N\left(\mathrm{C}^{18} \mathrm{O}\right)$ were calculated with a $\sim 2 \mathrm{~K}$ lower excitation temperature, or if the optical depth were calculated using a $0.5 \mathrm{~K}$ higher dust temperature. The latter applies to the highest $\tau(200 \mu \mathrm{m})$ values corresponding to $T_{\text {dust }} \sim 12 \mathrm{~K}$. b) The distribution of the $200 \mu \mathrm{m}$ optical depth over the area of highest optical depth. The dotted contour indicates the level $\tau(200 \mu \mathrm{m})=2 \times 10^{-3}$. In a) the points inside this contour are plotted with larger symbols. The solid contours show the distribution of the $\mathrm{C}^{18} \mathrm{O}$ column density. The highest contour is at $1.6 \times 10^{15} \mathrm{~cm}^{-3}$ and the others are each a factor of two below the previous one.

radiative transfer models will also be constructed for the far-infrared dust emission.

\subsection{Comparison of FIR and molecular line maps and column densities}

Figure 14 shows the distribution of FIR surface brightness relative to the intensity of the $\mathrm{C}^{18} \mathrm{O}(1-0)$ lines. The $200 \mu \mathrm{m}$ intensity closely follows the $\mathrm{C}^{18} \mathrm{O}$ emission. The peak positions do coincide and the second $200 \mu \mathrm{m}$ 
maximum in the southeast as well as fainter extensions towards south and towards southwest have corresponding features in the line map. The $200 \mu \mathrm{m}$ emission extends, however, somewhat further in the north while the $\mathrm{C}^{18} \mathrm{O}$ is relatively stronger in the southwest. With respect to the $200 \mu \mathrm{m}$ peak the $\mathrm{C}^{18} \mathrm{O}(1-0)$ maximum is displaced slightly towards the southwest. Further towards the southwest, the $\mathrm{C}^{18} \mathrm{O}$ is again well traced by the FIR emission. The second $\mathrm{C}^{18} \mathrm{O}(1-0)$ peak at position $(4,-2)$ coincides with the second $200 \mu \mathrm{m}$ peak. There is, however, a third $200 \mu \mathrm{m}$ peak at $(1,-6)$ which does not have a direct counterpart in the molecular line emission. The peak corresponds to just one pixel above the diffuse background and could even be caused by a single extragalactic source. The $100 \mu \mathrm{m}$ peak traces the eastern extension of the $\mathrm{C}^{18} \mathrm{O}$ distribution. Apart from this there is a clear lack of correlation with molecular line emission, especially within the $\mathrm{C}^{18} \mathrm{O}$ core.

In Fig. $15 \mathrm{C}^{18} \mathrm{O}(1-0)$ and ${ }^{13} \mathrm{CO}(2-1)$ line areas are compared with the $100 \mu \mathrm{m}$ and $200 \mu \mathrm{m}$ surface brightness values. Of the two far-infrared bands $200 \mu \mathrm{m}$ is clearly better correlated with molecular material. While on a logarithmic scale the relation between $\mathrm{C}^{18} \mathrm{O}(1-0)$ and $200 \mu \mathrm{m}$ emission is approximately linear, the ${ }^{13} \mathrm{CO}(2-1)$ intensity becomes saturated beyond $S(200 \mu \mathrm{m})=50 \mathrm{MJy} \mathrm{sr}^{-1}$. Based on IRAS observations Laureijs et al. (1995) calculated the difference $\Delta I_{100}=I(100 \mu \mathrm{m})-I(60 \mu \mathrm{m}) / 0.21$. The $60 \mu \mathrm{m}$ emission traces the diffuse outer parts of the cloud and the correlation with the ${ }^{13} \mathrm{CO}$ core was found to be better for $\Delta I_{100}$ than for the original $100 \mu \mathrm{m}$. The $100 \mu \mathrm{m}$ observations are affected by this warmer emission and this causes the poor correlation. The colour temperatures south of the L183 core could also be biased due to diffuse material the presence of which is evident from the ${ }^{12} \mathrm{CO}$ maps (Fig. 7).

In Fig. 16, the $\mathrm{C}^{18} \mathrm{O}(1-0)$ column density (see Fig. 13 and Sect. 4) is compared with the dust optical depth at $200 \mu \mathrm{m}$. The figure confirms the good general correlation between the two. The $\mathrm{C}^{18} \mathrm{O}$ column density distribution may, however, be slightly more extended in the SE. This was already seen in the $\mathrm{C}^{18} \mathrm{O}(1-0)$ distribution. On the other hand, $200 \mu \mathrm{m}$ optical depth is relatively larger immediately south of the $(0,0)$ position.

Nevertheless, the linear relation does hold only at lower column densities. A linear least squares fit was made taking into account the uncertainty in both variables. For points with $\tau(200 \mu \mathrm{m})<5 \times 10^{-3}$ we get

$$
\frac{N\left(\mathrm{C}^{18} \mathrm{O}\right)}{10^{13} \mathrm{~cm}^{-2}}=(4.62 \pm 0.16) 10^{4} \tau(200 \mu \mathrm{m})-(1.5 \pm 1.0) \text {. }
$$

Points at $\tau(200 \mu \mathrm{m})<5 \times 10^{-5}$ were also discarded for this fit since the $\mathrm{C}^{18} \mathrm{O}$ column density estimates corresponding to these points are no longer reliable. When $\tau(200 \mu \mathrm{m})$ exceeds $\sim 5 \times 10^{-3}$ the $\mathrm{C}^{18} \mathrm{O}$ column density is significantly below the prediction based on lower optical depths. The $\mathrm{C}^{18} \mathrm{O}$ emission could be reduced by depletion of gas phase molecules, a large drop in the excitation temperature towards the cold core or by the effect of optical depth.

\section{CO depletion in the cloud core}

The LTE analysis indicated $\mathrm{C}^{18} \mathrm{O}(1-0)$ optical depths below one. In the Monte Carlo model, the optical depth towards the cloud centre averaged over the telescope beam was 0.56 . These results were based on the ${ }^{13} \mathrm{CO}(1-0)$ and $\mathrm{C}^{18} \mathrm{O}(1-0)$ observations. Since the emission of the observed lines may come from different parts of the cloud, the determination of the optical depth remains uncertain to some extent. In the positions close to the cloud centre where $\mathrm{C}^{18} \mathrm{O}(2-1)$ was observed LTE analysis based on the two $\mathrm{C}^{18} \mathrm{O}$ lines gave somewhat higher estimates for the optical depth, $\tau \lesssim 1.0$. The optical depth is insufficient to cause any significant saturation of the $\mathrm{C}^{18} \mathrm{O}$ line and will not affect the accuracy of the column density estimates in Fig. 16.

Based on the LTE analysis, the excitation temperature was rather uniform over the cloud. Compared with other parts of the cloud the excitation temperature towards the cloud centre was lower by no more than $1 \mathrm{~K}$. The column density estimates were based on an average value of $8.4 \mathrm{~K}$. In Fig. 16, the vertical arrow indicates the change in the estimated column density if excitation temperature is reduced from a value $8.8 \mathrm{~K}$ to a value of $7.0 \mathrm{~K}$. It is clear that even a drop of $\sim 2 \mathrm{~K}$ is insufficient to explain the relatively low $\mathrm{C}^{18} \mathrm{O}$ column density estimates at the position of high values of $\tau(200 \mu \mathrm{m})$.

The horizontal arrow in Fig. 16 indicates the shift in the value of $\tau(200 \mu \mathrm{m})$ when a value of optical depth is calculated with $T_{\text {dust }}=12.5 \mathrm{~K}$ instead of $12.0 \mathrm{~K}$. Incorrect dust temperatures are, however, not likely to explain the relative drop of the $\mathrm{C}^{18} \mathrm{O}$ intensity. Firstly, the error should be $\sim 1 \mathrm{~K}$ or more at $12 \mathrm{~K}$ to bring the deviating points onto the fitted line even if the temperature were not changed outside the core. Errors in temperatures could be caused either by calibration or the background subtraction. If all $100 \mu \mathrm{m}$ surface brightness values are multiplied by a constant, all $\tau(200 \mu \mathrm{m})$ estimates would be scaled with a factor that has very weak temperature dependence. An incorrect background subtraction, on the other hand, mostly affects the high temperatures which correspond to low surface brightness values. As an example, let us consider two dust temperatures, $12 \mathrm{~K}$ and $15 \mathrm{~K}$. After the background subtraction, in L183 these typically correspond to $100 \mu \mathrm{m}$ surface brightness values of $7 \mathrm{MJy} \mathrm{sr}^{-1}$ and $3 \mathrm{MJy} \mathrm{sr}^{-1}$. If we subtract $0.5 \mathrm{MJy} \mathrm{sr}^{-1}$ from the $100 \mu \mathrm{m}$ measurements the colour temperatures would drop to $11.85 \mathrm{~K}$ and $14.45 \mathrm{~K}$ and the corresponding $200 \mu \mathrm{m}$ optical depth estimates would increase by $8 \%$ and $20 \%$, respectively. The net change is only some $12 \%$ i.e. much less than the effect seen in Fig. 16.

Therefore, it seems that $\mathrm{C}^{18} \mathrm{O}$ depletion remains the most probable cause for the decreasing $N\left(\mathrm{C}^{18} \mathrm{O}\right) / \tau(200 \mu \mathrm{m})$ ratio. In cloud cores the $\mathrm{CO}$ molecules freeze out onto the surfaces of the cold dust grains. Direct evidence of the process is provided by the observations of the molecular ice features (e.g. Tielens et al. 1991). As the result the properties of the dust 
grains are altered and this may explain some of the colour temperature variations observed in L183. In the centre of L183 the visual extinction exceeds $10^{\mathrm{m}}$ and $\mathrm{CO}$ depletion has been detected in other clouds at similar extinction values. Kramer et al. (1999) and Bergin et al. (2001) have detected $\mathrm{CO}$ depletion in the cloud IC 5146. In that cloud the relative $\mathrm{C}^{18} \mathrm{O}$ intensity decreases already below $A_{V}=10^{\mathrm{m}}$. Similarly in the cloud L977 a drop in $\mathrm{C}^{18} \mathrm{O}$ intensity attributed to depletion takes place at visual extinction $A_{V} \sim 10^{\mathrm{m}}$ (Alves et al. 1999). In L183 our models predicted central density close to $10^{5} \mathrm{~cm}^{-3}$ for the spherical model with a radial density profile $\sim r^{-1.5}$. This density is probably exceeded in the cold core and e.g. in L1544 CO depletion was associated with densities above $10^{5} \mathrm{~cm}^{-3}$ (Caselli et al. 1998).

Based in Fig. 16, the depletion factor is $\sim 1.5$ in the centre of L183 where the dust temperature is close to $12 \mathrm{~K}$. This is in perfect agreement with the findings of Kramer et al. (1999) who have derived the depletion factor for IC5146 as a function of dust temperature.

\section{DCO+ in the cold cloud core}

$\mathrm{H}^{13} \mathrm{CO}+$ and $\mathrm{DCO}+$ are concentrated close to the $200 \mu \mathrm{m}$ maximum. The peaks of the intensity distributions of both species are very close to the position of the continuum source, within $\sim 2^{\prime}$. While the $200 \mu \mathrm{m}$ emission continues to be strong towards the $100 \mu \mathrm{m}$ peak both $\mathrm{H}^{13} \mathrm{CO}+$ and $\mathrm{DCO}+$ are restricted to a narrow ridge running in the north-south direction. The distribution is therefore clearly different from either $\mathrm{C}^{18} \mathrm{O}$ or $200 \mu \mathrm{m}$, although close to position $(0,0)$ the $200 \mu \mathrm{m}$ emission is also elongated in the north-south direction. More importantly, no $\mathrm{H}^{13} \mathrm{CO}+$ or $\mathrm{DCO}+$ emission is seen close to the $100 \mu \mathrm{m}$ maximum, while the area of strong $\mathrm{C}^{18} \mathrm{O}$ emission covers also that position. At the position $\left(4.5^{\prime},-2.5^{\prime}\right)$ we get $2 \sigma$ upper limits of $0.10 \mathrm{~K}$ for $\mathrm{H}^{13} \mathrm{CO}+(1-0)$ and $0.20 \mathrm{~K}$ for $\mathrm{DCO}+(2-1)$ main beam temperature.

Figure 17 shows the $\mathrm{DCO}+$ distribution in relation to $\mathrm{C}^{18} \mathrm{O}$. Both peak in the same region around position $(0,0)$ and the small extension from this position towards the west can be interpreted to correspond to a similar feature seen in $\mathrm{C}^{18} \mathrm{O}$. However, at the exact position of the $\mathrm{DCO}+$ maximum, the $\mathrm{C}^{18} \mathrm{O}$ map shows a very slight depression, and in the south the two species are clearly anticorrelated, with stronger $\mathrm{C}^{18} \mathrm{O}$ emission seen on each side of the $\mathrm{DCO}+$ ridge.

The $100 \mu \mathrm{m}$ emission peaks some $5^{\prime}$ east of the $\mathrm{DCO}+$ maximum and even in details the distribution is completely unrelated to the $\mathrm{DCO}+$ emission. The correlation between $\mathrm{DCO}+$ and $200 \mu \mathrm{m}$ emission is not very good either. Both peak close to position $(0,0)$ but compared with the FIR emission the DCO+ maximum is shifted one arcminute to the west. Apart from a similar shift the correlation is good north of the centre position. In the south the distributions of $\mathrm{DCO}+$ and $200 \mu \mathrm{m}$ are different. As already mentioned, no $\mathrm{DCO}+$ emission was seen close to the eastern FIR peak. The DCO+ map does not quite extend to the southern $200 \mu \mathrm{m}$ peak at position $(1,-6)$ but between the northern and southern $200 \mu \mathrm{m}$ peaks the $\mathrm{DCO}+$ ridge goes over a region with relatively low FIR emission.

In Fig. 18a we compare the DCO+ distribution with the $200 \mu \mathrm{m}$ optical depth. Due to the dust temperature gradient, $\tau(200 \mu \mathrm{m})$ is shifted relative to the $200 \mu \mathrm{m}$ emission and coincides with the $\mathrm{DCO}+$ distribution. Around position $(0,0)$ and in the north, the correlation between $\tau(200 \mu \mathrm{m})$ and $\mathrm{DCO}+$ is very good. In the south, the $\mathrm{DCO}+$ follows roughly the dust optical depth distribution but the correlation is not perfect. The $\tau(200 \mu \mathrm{m})$ distribution is more extended SE of the centre position and the southern $\mathrm{DCO}+$ peak at $(1.5,-4)$ lies just north of the third $\tau(200 \mu \mathrm{m})$ peak, i.e. it does not follow dust distribution. The southern part of the $\mathrm{DCO}+$ ridge is outside the coldest dust core (Fig. 18b).

As the distributions of the $\mathrm{DCO}+$ and $\mathrm{H}^{13} \mathrm{CO}+$ are very similar, the same conclusions apply to the comparison of the $\mathrm{H}^{13} \mathrm{CO}+$ distribution with $\mathrm{CO}$ species and FIR emission. At the position $(0,0)$, the observed main beam temperatures are $1.5 \mathrm{~K}$ and $0.79 \mathrm{~K}$ for $\mathrm{DCO}+(2-1)$ and $\mathrm{H}^{13} \mathrm{CO}+(1-0)$ respectively. $\mathrm{DCO}+$ seems to be more concentrated in this core, but the difference is not significant when the difference in the beam size is taken into account. In the upper panel of Fig. 19 the $\mathrm{DCO}+$ and $\mathrm{H}^{13} \mathrm{CO}+$ intensities are compared along the line going through the two cores. The $\mathrm{DCO}+$ values were convolved to the resolution of the $\mathrm{H}^{13} \mathrm{CO}+$ observations. In the main core, the $\mathrm{H}^{13} \mathrm{CO}+$ is displaced to the north with respect to the $\mathrm{DCO}+$. In the southern core, the relative intensity of $\mathrm{H}^{13} \mathrm{CO}+$ is higher.

According to chemical models (Millar et al. 1989; Roberts \& Millar 2000) the ratio between DCO+ and $\mathrm{H}^{13} \mathrm{CO}+$ can be used as a tracer of gas temperature. The reaction

$\mathrm{H}_{3}^{+}+\mathrm{HD} \leftrightarrow \mathrm{H}_{2} \mathrm{D}^{+}+\mathrm{H}_{2}+\Delta E$

is strongly temperature dependent. At low temperatures the production of $\mathrm{H}_{2} \mathrm{D}^{+}$is enhanced and it reacts with $\mathrm{CO}$ to produce more $\mathrm{DCO}+$. The abundance ratio also depends, however, on other factors such as cloud age, electron density and the total abundance of neutrals (Anderson et al. 1999; Roberts \& Millar 2000). This prevents the use of the ratio $[\mathrm{DCO}+] /\left[\mathrm{H}^{13} \mathrm{CO}+\right]$ for direct temperature determination.

The observed change in the intensity of the $\mathrm{DCO}+$ and $\mathrm{H}^{13} \mathrm{CO}+$ lines indicates a higher gas temperature in the southern core. This is consistent with the difference in dust temperatures. Using the main beam antenna temperatures, the ratio of $\mathrm{DCO}+(2-1)$ and $\mathrm{H}^{13} \mathrm{CO}+(1-0)$ line areas increases from $\sim 1.0$ in the south to $\sim 1.7$ in the main core. The excitation states of $\mathrm{DCO}+$ and $\mathrm{H}^{13} \mathrm{CO}+$ should be very similar. The isothermal Monte Carlo model used to model the ${ }^{13} \mathrm{CO}$ and $\mathrm{C}^{18} \mathrm{O}$ lines was used to predict $\mathrm{DCO}+$ lines assuming an abundance of $5 \times 10^{-11}$. Compared with the model in Sect. 4 , the density needed to be increased almost a factor of two to produce lines 


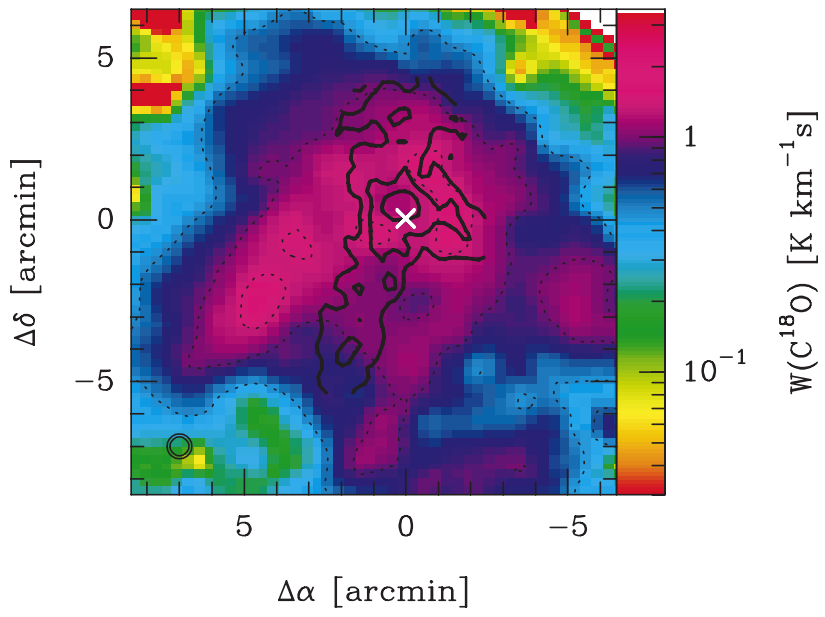

Fig. 17. Contours of $\mathrm{DCO}+(2-1)$ integrated line area on the map of integrated $\mathrm{C}^{18} \mathrm{O}(1-0)$ line area. The $\mathrm{DCO}+$ contours are at $0.2,0.4$, and $0.6 \mathrm{~K} \mathrm{~km} \mathrm{~s}^{-1}$. The dotted contours indicate $\mathrm{C}^{18} \mathrm{O}(1-0)$ intensity levels from 0.5 to $2.0 \mathrm{~K} \mathrm{~km} \mathrm{~s}^{-1}$ at intervals of $0.5 \mathrm{~K} \mathrm{~km} \mathrm{~s}^{-1}$. The maps are presented at their original resolution. The beam sizes ( $46^{\prime \prime}$ for $\mathrm{C}^{18} \mathrm{O}$ and $35^{\prime \prime}$ for $\mathrm{DCO}+$ ) are indicated with circles at the lower left corner.

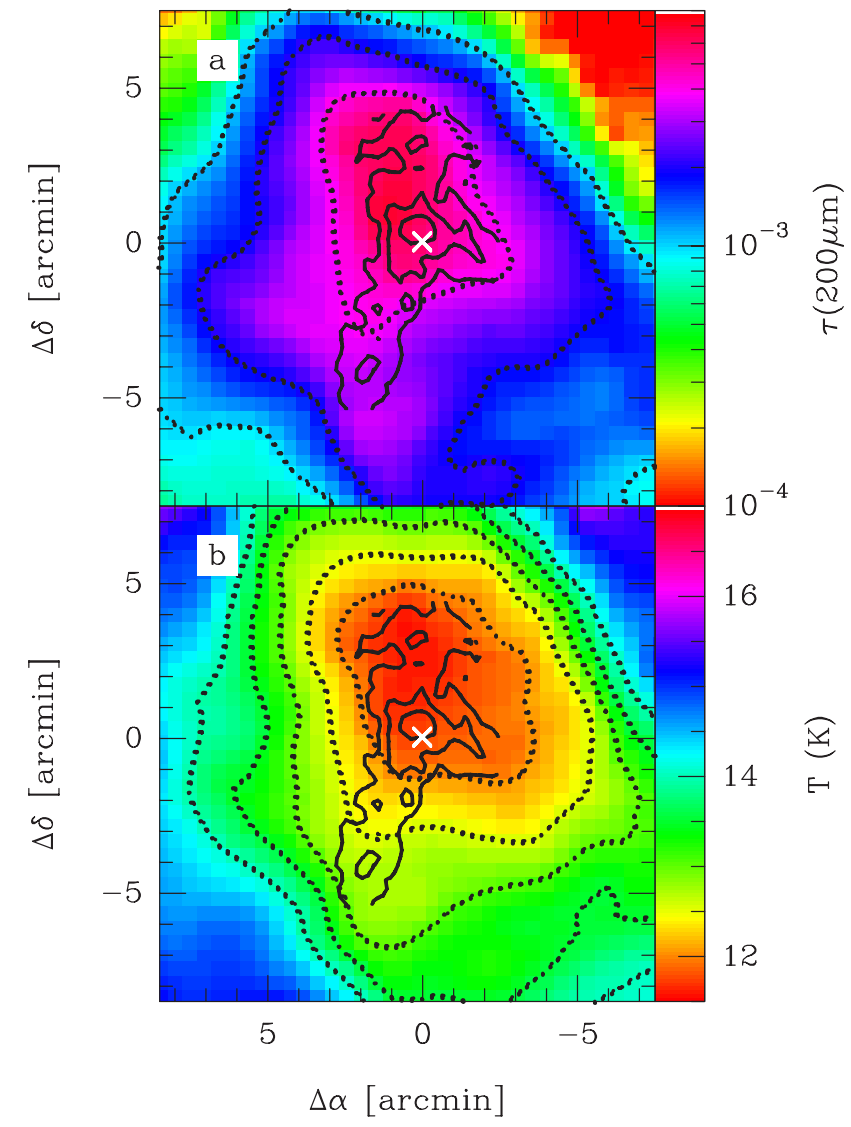

Fig. 18. a) DCO $+(2-1)$ contours on the map of $200 \mu \mathrm{m}$ optical depth. The solid contours show the $\mathrm{DCO}+$ distribution and are drawn at $0.2,0.4$, and $0.6 \mathrm{~K} \mathrm{~km} \mathrm{~s}^{-1}$. The dotted contours indicate values $1 \times 10^{-3}, 2 \times 10^{-3}$ and $4 \times 10^{-3}$ of $200 \mu \mathrm{m}$ optical depth. b) $\mathrm{DCO}+(2-1)$ contours on the map of dust colour temperature. The solid $\mathrm{DCO}+$ contours are drawn at $0.3,0.5,0.7$ and $0.9 \mathrm{~K} \mathrm{~km}^{-1}$. The dotted contours correspond to dust temperatures from 12 to $14 \mathrm{~K}$ at steps of $0.5 \mathrm{~K}$. The maps are presented at their original resolution.
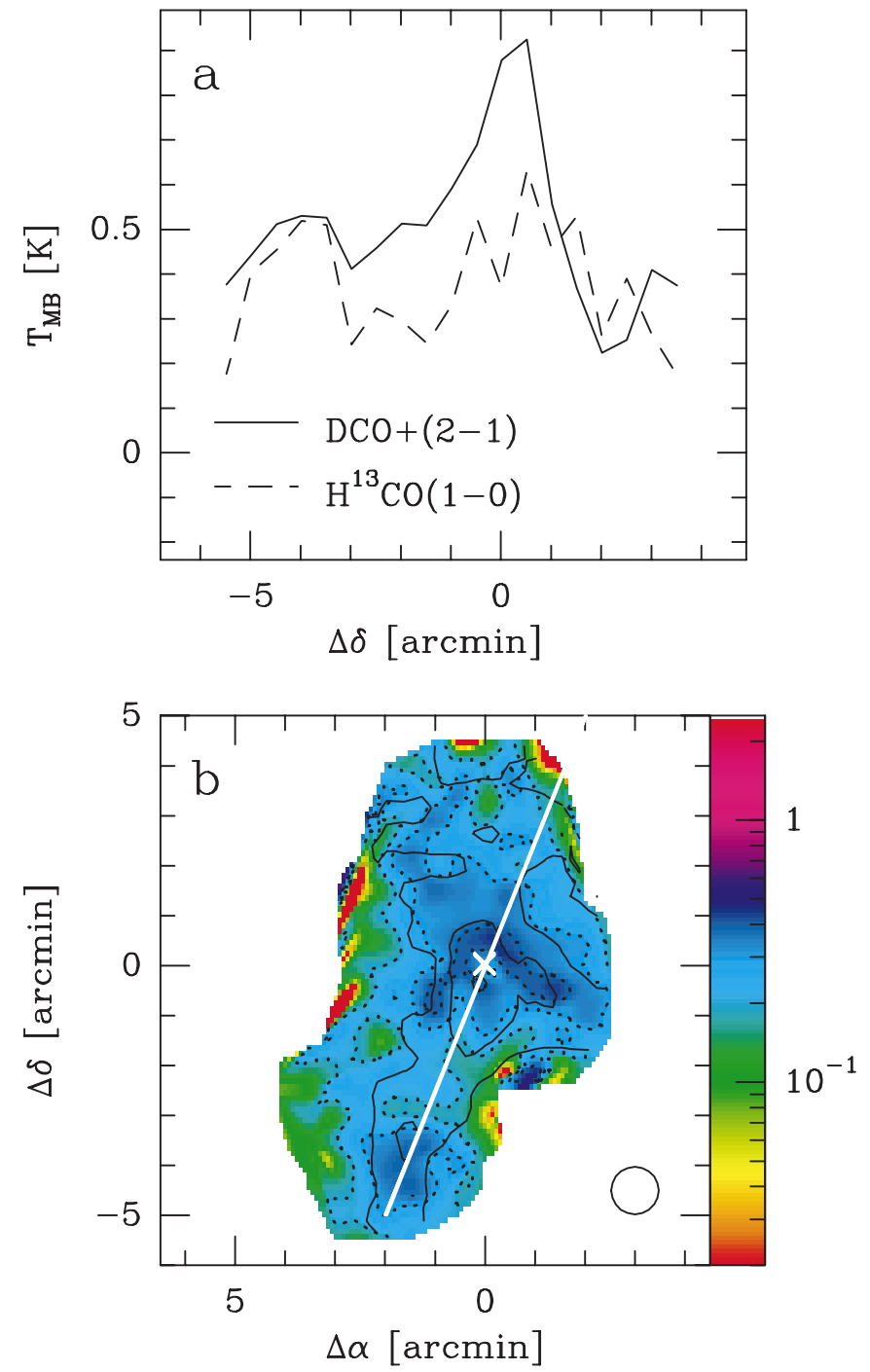

Fig. 19. a) The $\mathrm{DCO}+(2-1)$ (solid line) and $\mathrm{H}^{13} \mathrm{CO}+(1-0)$ (dashed line) intensities along the line going through the two cores detected in these lines. b) $\mathrm{DCO}+(2-1)$ contours (solid lines) over-plotted on the $\mathrm{H}^{13} \mathrm{CO}+(1-0)$ map. The DCO+ contours levels are the same as in the previous figures. The dotted lines indicate $\mathrm{H}^{13} \mathrm{CO}+(1-0)$ values $0.2,0.3$ and $0.4 \mathrm{~K} \mathrm{~km} \mathrm{~s}^{-1}$. The $\mathrm{DCO}+$ data are convolved to the resolution of $\mathrm{H}^{13} \mathrm{CO}+$ observations.

$T_{\mathrm{A}}(J=1-0) \sim 0.8 \mathrm{~K}$, in which case the line ratio was $T(1-0) / T(2-1) \sim 2$. Assuming column density is directly proportional to observed intensity and using a value of 66 for the ratio ${ }^{12} \mathrm{C} /{ }^{13} \mathrm{C}$ (Langer \& Penzias 1993), we obtain values of 0.051 and 0.030 for the $\mathrm{DCO}+$ to $\mathrm{HCO}+$ abundance ratio in the two cores. The ratios indicate gas temperatures $\sim 10 \mathrm{~K}$ or less, depending on the set of reaction constants used (Millar et al. 1989; Roberts \& Millar 2000; see also Loren et al. 1990). The difference between the northern and southern cores corresponds to a temperature change of at least $2 \mathrm{~K}$. 


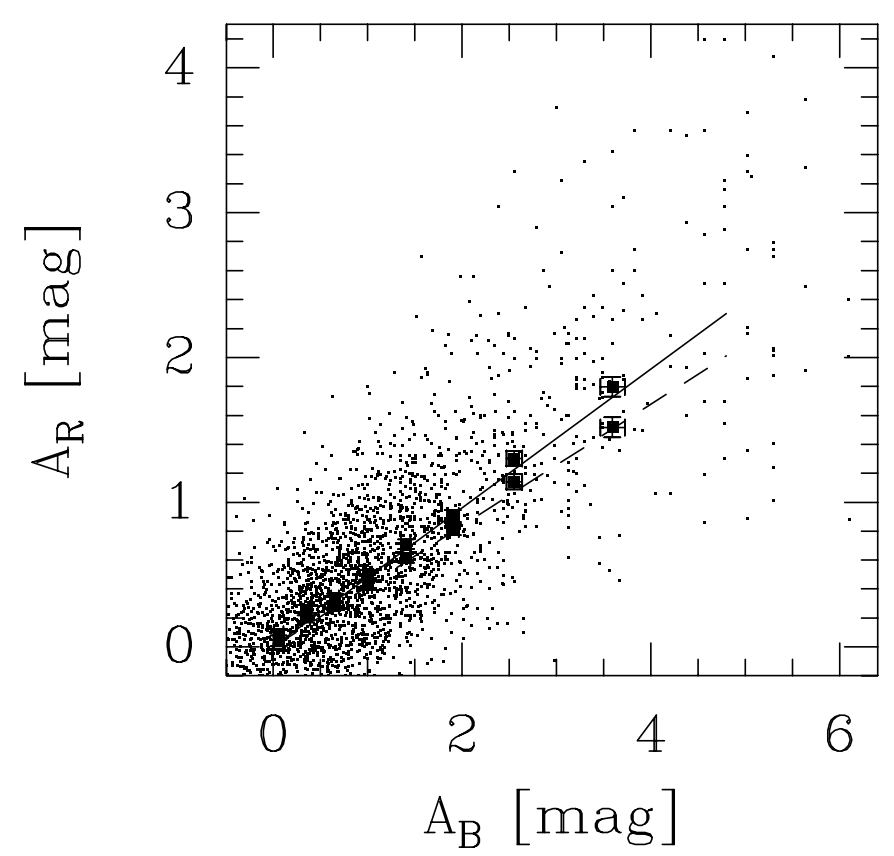

Fig. 20. The relation between $B$-band and $R$-band extinction as derived from the starcounts. The lower points with errorbars are obtained by first averaging the $R$-band counts in areas with given number of $B$-band stars. The dashed line is the least squares line fitted to these. The upper points denote averages of $A_{R}$ for given $A_{B}$ and the dots correspond to star count averaged over circle with radius $1.7^{\prime}$. The solid line is the least squares fit to these points and has a slope of 0.48 .

\section{Comparison of optical extinction with FIR dust and $\mathrm{CO}$ column densities}

In Fig. 20 we plot $A_{R}$ as a function of $A_{B}$, as derived from the starcounts (see Sect. 2.3). The dots denote individual extinction values obtained by averaging the counts in each grid point over a circle with radius $1.7^{\prime}$. In the averaging the weights were proportional to the area inside the circle which was, however, only slightly larger than original $2.8^{\prime} \times 2.8^{\prime}$ square used in the star counts.

We first calculated the average number of $R$-band stars in those $2.8^{\prime} \times 2.8^{\prime}$ squares with a given number of $B$-band stars. The average counts in these areas were transformed into extinction values. The relation with statistical errors is shown in Fig. 20 (dashed line). Linear least squares fit gives a relation $A_{R}=(0.42 \pm 0.02) A_{B}$. The fit takes into account uncertainty in both $A_{B}$ and $A_{R}$. If the intercept were included in the fit its value would be only $\sim 0.03$. If we calculate the average of the $A_{R}$ values at all grid points with a given $A_{B}$ (instead of first calculating the average number of stars) the derived relation becomes steeper, with a slope of $0.48 \pm 0.02$. The value is still somewhat lower than the value $\sim 0.56$ for the extinction law with $R_{V}=A_{B} / E_{B-V}=3.1$. The fit is shown as a solid line in the figure. The least squares line fitted directly to $\left(A_{B}, A_{R}\right)$ points gives also a slope of 0.48 . Considering the possible systematic errors of $\sim 10 \%$ of our extinction values, due to uncertainties in the calibration of $\log N(m)$ vs. $m$ slopes, our $A_{R} / A_{B}$ ratio is compatible with the standard reddening law $\left(R_{V}=3.1\right)$. On the other hand, it differs significantly from the value of $A_{R} / A_{B}=0.66$ valid for the case of $R_{V}=5.0$ as derived for the "outer-cloud dust" (Mathis et al. 1990).

In the centre of L183 there were a few starcount squares where no stars were seen and for Fig. 20 the extinction was calculated using a value of 0.5 stars per square. The values, $A_{R} \approx 5^{\mathrm{m}}$, still only represent a lower limit of the true extinction. In the most opaque area of 95 sq arcmin, four stars were detected in the $R$-band and this translates into an extinction of $5.3^{\mathrm{m}}$. In the $B$-band, no stars were seen in this area while a single star would correspond to an extinction of $6.8^{\mathrm{m}}$. Therefore, it is likely that at the very centre the extinction is at least $A_{V} \sim 10^{\mathrm{m}}$.

Next $A_{R}$ is compared with the hydrogen column density estimate derived from the $\mathrm{C}^{18} \mathrm{O}(1-0)$ observations (Fig. 13) and the $200 \mu \mathrm{m}$ optical depth (Fig. 6). The data are convolved to the resolution $2.8^{\prime}$ corresponding to the grid size used in the starcounts. The results are shown in Figs. 21 and 22 .

The $\mathrm{C}^{18} \mathrm{O}$ column density and the extinction are clearly correlated. The $\mathrm{C}^{18} \mathrm{O}$ depletion seen in Fig. 16 is not visible, however, as the centre region where no $R$ band stars were detected is not included in the figure. This limits the dynamical range available. Furthermore, at low column densities the noise increases rapidly due to the decreasing signal-to-noise ratio of the $\mathrm{C}^{18} \mathrm{O}$ spectra. The maximum extinction corresponding to one star per grid pixel area was $\sim 4^{\mathrm{m}}$. For the plot the star counts were again averaged over a circle with radius $1.7^{\prime}$ but points with $A_{R}>4^{\mathrm{m}}$ are omitted in the figure. The least squares line is

$$
A_{R} / \mathrm{mag}=(3.4 \pm 0.2) \times 10^{-15} N\left(\mathrm{C}^{18} \mathrm{O}\right)+(0.1 \pm 0.1)
$$

With the earlier relation between $A_{\mathrm{R}}$ and $A_{\mathrm{B}}$ and the assumed value of $R=3.1$ this translates to $N\left(\mathrm{C}^{18} \mathrm{O}\right) / \mathrm{cm}^{-2}=1.9 \times 10^{14} A_{V}-4 \times 10^{13}$. The slope is similar to the value found in regions of high extinction in Taurus and Ophiuchus by Frerking et al. (1982) but steeper than the corresponding value for the lower range $2^{\mathrm{m}}<A_{V}<4^{\mathrm{m}}$ where the value in Taurus was $0.7 \times 10^{14} \mathrm{~cm}^{-2} \mathrm{mag}^{-1}$. The slopes determined in L977 and IC5146 for $A_{V} \lesssim 10^{\mathrm{m}}$ (Alves et al. 1999) are rather similar, $\sim 2 \times 10^{14} \mathrm{~cm}^{-2} \mathrm{mag}^{-1}$. Also Harjunpää et al. (1996) found a similar slope in Corona Australis while in the Coalsack the value was only $\sim 1.2 \times 10^{14} \mathrm{~cm}^{-2} \mathrm{mag}^{-1}$. Note that if we assume standard extinction (Rieke \& Lebofsky 1985) to transform $A_{R}$ directly to $A_{V}$ our slope increases to $2.2 \times 10^{14} \mathrm{~cm}^{-2} \mathrm{mag}^{-1}$.

Within the statistical uncertainty of the starcounts, the extinction map agrees well with $\tau(200 \mu \mathrm{m})$. The dots in Fig. 22 correspond to the star counts on the original grid. The filled squares with error bars are averages for each discrete value of $A_{R}$. The least squares line based on the interval $1.0^{\mathrm{m}}<A_{R}<4.0^{\mathrm{m}}$ is

$$
A_{R} / \mathrm{mag}=(2.2 \pm 0.3) \times 10^{3} \tau(200 \mu \mathrm{m})+(0.2 \pm 0.2) .
$$



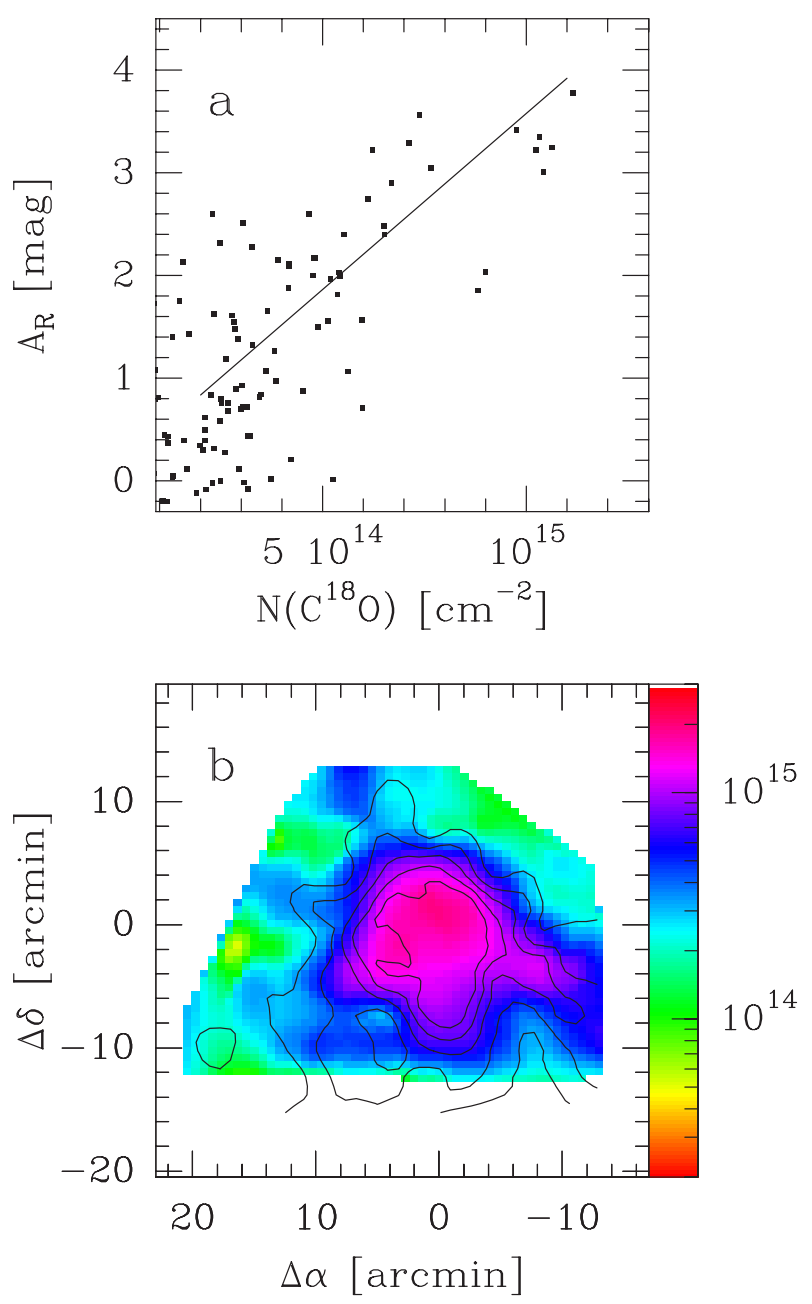

Fig. 21. a) $R$-band extinction vs. $\mathrm{C}^{18} \mathrm{O}$ column density. b) $R$ band extinction contours drawn on the $\mathrm{C}^{18} \mathrm{O}$ column density map. The contours of extinction are drawn between $1^{\mathrm{m}}$ and $5^{\mathrm{m}}$ at intervals of $1^{\mathrm{m}}$. The $\mathrm{C}^{18} \mathrm{O}$ column densities have been convolved to the resolution of the extinction map, $\sim 2.8^{\prime}$.

The relation can be used to derive an extrapolated extinction value for the centre region where no stars were detected. In the centre there are several $200 \mu \mathrm{m}$ pixels with $\tau(200 \mu \mathrm{m}) \sim 6 \times 10^{-3}$. According to the previous relation, this corresponds to extinction $A_{R} \approx 13^{\mathrm{m}}$ and visual extinction of the order of $17^{\mathrm{m}}$. This clearly exceeds the previous estimates, and is based on the sharp increase of the $200 \mu \mathrm{m}$ optical depth in the cold cloud core.

Assuming a standard extinction law $\left(R_{V}=3.1\right)$ between $V$ - and $R$-bands, from Eq. (5) we obtain $\tau(200 \mu \mathrm{m}) / A_{V}=3.4 \times 10^{-4} \mathrm{mag}^{-1}$. For the cloud centre, the ratio increases to $3.8 \times 10^{-4} \mathrm{mag}^{-1}$ but is still below the value of $5.3 \times 10^{-4} \mathrm{mag}^{-1}$ found in the Thumbprint Nebula (Lehtinen et al. 1998). In L183, the comparison between $100 \mu \mathrm{m}$ optical depth and visual extinction gives a ratio $2.1 \times 10^{-3} \mathrm{mag}^{-1}$ which is similar to the value in the Thumbprint Nebula.

For a value of $\tau(200 \mu \mathrm{m})=1.0 \times 10^{-3}$, the previous relations between $\tau(200 \mu \mathrm{m})$ and $A_{R}$ and between $A_{R}$ and $\mathrm{C}^{18} \mathrm{O}$ column density predict a column density of
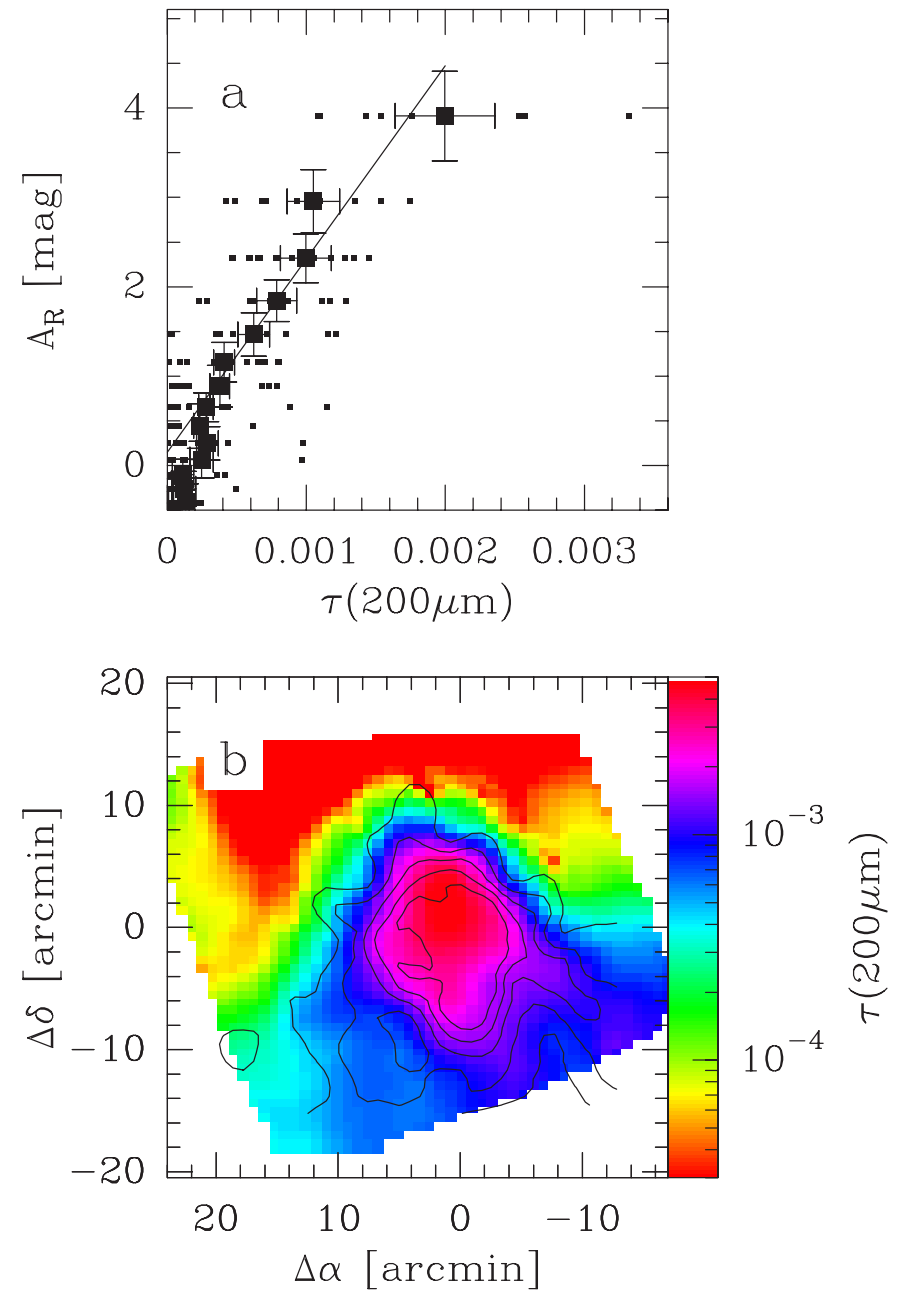

Fig. 22. a) $R$-band extinction vs. $200 \mu \mathrm{m}$ optical depth. b) $R$ band extinction contours on the map of $\tau(200 \mu \mathrm{m})$ which has been convolved to a resolution of $2.8^{\prime}$. The extinction contours are drawn between $1^{\mathrm{m}}$ and $5^{\mathrm{m}}$ at intervals of $1^{\mathrm{m}}$.

$N\left(\mathrm{C}^{18} \mathrm{O}\right)=6.6 \times 10^{14} \mathrm{~cm}^{-2}$. Directly using the relation between $\tau(200 \mu \mathrm{m})$ and $\mathrm{N}\left(\mathrm{C}^{18} \mathrm{O}\right)$ (Eq. (2)) we would obtain a lower value, $4.5 \times 10^{14} \mathrm{~cm}^{-2}$. One must remember, however, that Eq. (2) was determined over a much larger range of optical depths and consequently that relation may already be affected by the $\mathrm{C}^{18} \mathrm{O}$ depletion.

\section{Conclusions}

The main conclusions drawn from the comparison of farinfrared data and molecular line observations in L183 are:

- The $100 \mu \mathrm{m}$ and $200 \mu \mathrm{m}$ emission clearly have different distributions.

- The $200 \mu \mathrm{m}$ maximum indicates the position of a core where either the dust temperature becomes very low or the dust properties have changed. The colour temperature determined from the $100 \mu \mathrm{m}$ and $200 \mu \mathrm{m}$ observations with an emissivity law of $\nu^{2}$ is $\lesssim 12 \mathrm{~K}$. The dust optical depth peaks at the same position.

- Of the molecular lines, the optically thin $\mathrm{C}^{18} \mathrm{O}$ best traces the dust distribution. Although better 
correlated with the $200 \mu \mathrm{m}$ surface brightness it shows strong emission at the locations of both the $100 \mu \mathrm{m}$ and $200 \mu \mathrm{m}$ maxima. The optically thick ${ }^{12} \mathrm{CO}$ and ${ }^{13} \mathrm{CO}$ lines peak south of the dust cores.

- $\mathrm{C}^{18} \mathrm{O}$ is depleted in the cloud centre. The depletion factor is $\sim 1.5$ in the core where dust temperature is close to $12 \mathrm{~K}$.

- Relative to each other, the $\mathrm{DCO}+$ and $\mathrm{H}^{13} \mathrm{CO}+$ lines show very similar distributions. Their distribution is completely different from any of the observed CO lines but a good correlation does exist with the $200 \mu \mathrm{m}$ optical depth.

- The estimated mass within $10^{\prime}$ of the $(0,0)$ position was $25 M_{\odot}$, based on the $200 \mu \mathrm{m}$ optical depths and $\sim 40 M_{\odot}$ based on the $\mathrm{C}^{18} \mathrm{O}$ column densities.

- The $100 \mu \mathrm{m}$ surface brightness is not well correlated with the extinction. A linear relation does exist between $A_{\mathrm{R}}$ and dust optical depth at $200 \mu \mathrm{m}$. Based on this correlation we derive for the cloud centre an extinction $A_{V} \sim 17^{\mathrm{m}}$.

Acknowledgements. Star counts were performed by J. Piironen (Helsinki University Observatory) using observations made by O. Pizarro (ESO) on the request of G. Schnur (RuhrUniversität Bochum) for this project. The ISOPHOT project was funded by Deutsches Zentrum für Luft- und Raumfahrt (DLR), the Max-Planck-Gesellschaft, the Danish, British and Spanish Space Agencies and several European institutes. M.J., K.M., and K.L acknowledge the support of the Academy of Finland Grant No. 1011055.

\section{References}

Alves, J., Lada, C. J., \& Lada, E. A. 1999, ApJ, 515, 265

Anderson, I. M., Caselli, P., Haikala, L. K., \& Harju, J. 1999, A\&A, 347, 983

Bahcall, J. N., Ratnatunga, K. U., Buser, R., Fenkart, R. P., \& Spaenhauer, A. 1985, ApJ, 299, 616

Bahcall, J. N., \& Soneira, R. M. 1980, ApJS, 44, 73

Becker, W., \& Fenkart, R. 1976, Photometric Catalogue of Stars in Selected Areas and other Fields in the RGUSystem, vol. 1, Basel: Astron. Inst. Univ. Basel

Bergin, E. A., Ciardi, D. R., Lada, C. J., Alves, J., \& Lada, E. A. 2001, ApJ, submitted [astro-ph 0103521]

Bergin, E. A., Langer, W. D., \& Goldsmith, P. F. 1995, ApJ, 441,222

Bergin, E. A., Snell, R. L., \& Goldsmith, P. F. 1996, ApJ, 460, 343

Bernard, J.-P., Abergel, A., Ristorcelli, I., et al. 1999, A\&A, 347,640

Bettens, R. P. A, Lee, H.-H., \& Herbst, E. 1995, ApJ, 443, 664

Bohlin, R. C., Savage, B. D., \& Drake, J. F. 1978, ApJ, 224, 132

Bok, B. J. 1956, AJ, 61, 309

Boulanger, F., Abergel, A., Bernard, J. P., et al. 1996, A\&A, 312,256

Cambrésy, L, Boulanger, F., Lagache, G., \& Stepnik, B. 2001, A\&A, submitted [astro-ph 0106507]
Caselli, P., Walmsley, C. M., Tafalla, M., Dore, L., \& Myers, P. C. 1999, ApJ, 532, L165

Caselli, P., Walmsley, C. M., Terzieva, R., \& Herbst, E. 1998, ApJ, 499, 234

Clark, F. O., Laureijs, R. J., \& Prusti, T. 1991, ApJ, 371, 602

Désert, F.-X., Boulanger, F., \& Puget, J. L. 1990, A\&A, 237, 215

Dickens, J. E., Irvine, W. M., Snell, R. L., et al. 2000, ApJ, 542,870

Franco, G. 1989, A\&A, 223, 313

Frerking, M. A., Langer, W. D., \& Wilson, R. W. 1982, ApJ, 262,590

García-Lario, P. 2000, 2nd post-operations cross-calibration report v. 1.0 http://www.iso.vilspa.esa.es/users/ expl_lib/ISO/2nd_status_report_ext.ps.gz

Gibb, A. G., \& Little, L. T. 1998, MNRAS, 295, 299

Guélin, M., Langer, W. D., \& Wilson, R. W. 1982, A\&A, 107, 107

Guélin, M., Langer, W. D., Snell, R. L., \& Wootten, H. A. 1977, ApJ, 217, L165

Harjunpää, P., \& Mattila, K. 1996, A\&A, 305, 920

Herbst, E., \& Leung, C. M. 1989, ApJS, 69, 271

Herbst, E., \& Leung, C. M. 1990, A\&A, 233, 177

Juvela, M. 1997, A\&A, 322, 943

Kessler, M. F., Steinz, J. A., Anderegg, M. E., et al. 1996, A\&A, 315, L27

Kramer, C., Alves, J., Lada, C. J., et al. 1999, A\&A, 342, 257

Laureijs, R. J., Clark, F. O., \& Prusti, T. 1991, ApJ, 372, 185

Laureijs, R. J., Fukui, Y., Helou, G., et al. 1995, ApJS, 101, 87

Laureijs, R. J., Haikala, L., Burgdorf, M., et al. 1996, A\&A, 315, L317

Lee, H.-H., Bettens, R. P. A., \& Herbst, E. 1996, A\&AS, 119, 111

Lehtinen, K., Lemke, D., Mattila, K., \& Haikala, L. K. 1998, A\&A, 333, 702

Lehtinen, et al. 2000, in ISO Surveys of a Dusty Universe, Lecture Notes in Physics, ed. D. Lemke, M. Stickel, \& K. Wilke (Springer), 317

Lee, H.-H., Herbst, E., Pineau des Forêts, G., et al. 1996, A\&A, 311,690

Lemke, D., Klaas, U., Abolins, J., et al. 1996, A\&A, 315, L64

Langer, W. D., \& Penzias, A. A. 1993, ApJ, 408, 539

Leung, C. M., Herbst, E., \& Huebner, W. F. 1984, ApJS, 56 , 231

Liljeström, T. 1991, A\&A, 244, 483

Loren, R. B., Wootten, A., \& Wilking, B. A. 1990, ApJ, 365, 269

Mathis, J. S. 1990, ARAA, 28, 37

Mattila, K. 1979, A\&A, 78, 253

Meny, C., Serra, G., Lamarre, J. M., et al. 2000, ESA-SP 455, 105

Millar, T. J., Bennett, A., \& Herbst, E. 1989, ApJ, 340, 906

Millar, T. J., Farquhar, P. R. A., \& Willacy, K. 1996, A\&AS, 121,139

Ossenkopf, V. 1993, ApJ, 280, 617

Padoan, P., Juvela, M., Bally, J., \& Nordlund 2000, ApJ, 529, 259

Pratap, P., Dickens, J. E., Snell, R. L., et al. 1997, ApJ, 486, 862

van Rhijn, P. J. 1929, Publ. Astron. Lab. Groningen, No. 43

Rieke, G. H., \& Lebofsky, M. J. 1985, ApJ, 288, 618

Roberts, H., \& Millar, T. J. 2000, A\&A, 361, 388 
Snell, R. L. 1981, ApJS, 45, 121

Snell, R. L., Schloerb, F. P., Young, J. S., et al. 1981, ApJ, 244,45

Snyder, L. E., Hollis, J. M., Buhl, D., \& Watson, W. D. 1977, ApJ, 218, 61

Stahler, S. W. 1984, ApJ, 281, 209

Stark, R., Wesselius, P. R., van Dishoeck, E. F., \& Laureijs, R. J. 1996, A\&A, 311, 282

Stepnik, B., Abergel, A., Bernard, J.-P., et al. 2001, A\&A, in press

Swade, D. A. 1989, ApJS, 71, 219

Swade, D. A. 1989, ApJ, 345, 828

Swade, D. A., \& Schloerb, F. P. 1992, ApJ, 392, 543

Tielens, A., Tokunaga, A., Geballe, T., \& Baas, F. 1991, ApJ, 381,181
Turner, B. E., Terzieva, R., \& Herbst, E. 1999, ApJ, 518, 699

Turner, B. E., Herbst, E., \& Terzieva, R. 2000, ApJS, 126, 427 Ungerechts, H., Walmsley, C. M., \& Winnewisser, G. 1980, A\&A, 88, 259

Ward-Thompson, D., Scott, P. F., \& Hills, R. E. 1994, MNRAS, 268, 276

Ward-Thompson, D., Kirk, J. M., Crutcher, R. M., et al. 2000, ApJ, 537, 135

Warin, S., Benayoun, J. J., \& Viala, Y. P. 1996, A\&A, 308, 535

Watson, W. D., Snyder, L. E., \& Hollis, J. M. 1978, ApJ, 222, L145

Wright, E. L. 1987, ApJ, 320, 818

Wootten, A., Loren, R. B., \& Snell, R. L. 1982, ApJ, 255, 160

Wootten, A., \& Loren, R. B. 1987, ApJ, 317, 220 